\title{
The Spherical Growth Series for Pure Artin Groups of Dihedral Type
}

\author{
by
}

\author{
Michihiko FuJII
}

\begin{abstract}
We consider the kernel of the natural projection from the Artin group of dihedral type $I_{2}(k)$ to the corresponding Coxeter group, which we call the pure Artin group of dihedral type. We present a rational function expression for the spherical growth series of the pure Artin group of dihedral type with respect to a natural generating set.

2010 Mathematics Subject Classification: Primary 20F36, 20F05, 20F10; Secondary 68R15. Keywords: Artin group of dihedral type, pure braid group, spherical growth series, Garside type normal form, automaton, automatic group.
\end{abstract}

\section{$\S 1$. Introduction}

For a finitely generated group $G$ with a given generating set $\Gamma$, we have the concept of the spherical growth series

$$
\mathcal{S}_{G, \Gamma}(t):=\sum_{n=0}^{\infty} \alpha_{n} t^{n}
$$

where $\alpha_{n}$ for $n \in \mathbb{N} \cup\{0\}$ is the number of elements in $G$ whose lengths with respect to $\Gamma$ are equal to $n$ (cf. [Mil] and [Sc]). The series $\mathcal{S}_{G, \Gamma}(t)$ provides a method to derive the combinatorial structure of $(G, \Gamma)$. In many cases, this series is known to be rational (cf. [Br], [C1], [C2], [Ca], [Ca-W], [dlH], [E], [E-IF-Z] and $[\mathrm{Fl}-\mathrm{P}])$. Rationality is usually proved by determining a particular geodesic representative for each element of $G$ with respect to $\Gamma$ and showing that the set of all such geodesics is recognized by deterministic, finite-state automata. In fact, Mairesse and Mathéus [M-M] constructed such automata for each Artin group $G_{I_{2}(k)}$ of dihedral type and obtained an explicit rational function expression for

Communicated by T. Ohtsuki. Received June 22, 2012. Revised February 12, 2013.

M. Fujii: Department of Mathematics, Graduate School of Science, Kyoto University, Kyoto 606-8502, Japan;

e-mail: mfujii@math.kyoto-u.ac.jp

(C) 2013 Research Institute for Mathematical Sciences, Kyoto University. All rights reserved. 
its spherical growth series with respect to the standard generating set $\Sigma$. (The rational function expression for the case $k=3$ is also given in Sabalka [Sab].)

In this paper, we consider the pure Artin group $P_{I_{2}(k)}$, which is the kernel of the projection from $G_{I_{2}(k)}$ to its Coxeter group, $\bar{G}_{I_{2}(k)}$. The group $P_{I_{2}(k)}$ is geometrically realized as the fundamental group of the complement of some arrangement of $k$ complex lines in $\mathbb{C}^{2}$, which has a natural generating set $A$ (cf. [R]). In particular, in the case $k=3, P_{I_{2}(3)}$ is the pure braid group with three strands, and $A$ is the standard generating set. Here, we present a rational function expression for the spherical growth series of $P_{I_{2}(k)}$ with respect to the generating set $A$.

Although the presentation of the group $P_{I_{2}(k)}$ over $A$ does not resemble that of $G_{I_{2}(k)}$ over $\Sigma$, the arguments similar to those used in [G] regarding the latter can also be applied to the case of $P_{I_{2}(k)}$. For example, noting the similar structures of the fundamental blocks considered here (defined in $\$ 2$ ) and Garside's generators, we can set up a correspondence between the sets of each, and using this correspondence, we can obtain a normal form for each element of $P_{I_{2}(k)}$ that is similar to Garside's normal form for an element of $G_{I_{2}(k)}$ (see Proposition 2.9). Then the procedure presented in [M-M] (the so-called 'suitable-spread procedure') can be applied to these normal forms. Specifically, we show that for any element $g$ of $P_{I_{2}(k)}$ that has a representative of a particular type (Type 3 defined in $\S 3$ ), all of the geodesic representatives of $g$ can be obtained by applying the suitable-spread procedure to its normal form (see Proposition 3.7). In this way, we can determine all of the geodesic representatives of this element. Moreover, even in the case that several geodesic representatives exist for such a given element of $P_{I_{2}(k)}$, we show that only one of these satisfies a certain necessary criterion, and hence the proper geodesic representative for that element too is uniquely determined (see Proposition 3.9). These geodesic representatives can be expressed as elements of the free monoid generated by a finite set $\Lambda$, and Proposition 3.9 presents a sufficient condition for the existence of deterministic, finite-state automata over $\Lambda$ that recognize these geodesic representatives. Then, in $\S 4$, we construct such automata explicitly. Next, by considering the structure of these automata, we derive an exact form of the rational function expression for the spherical growth series of $P_{I_{2}(k)}$ (see Theorem 5.7).

We define the positive monoid $P_{I_{2}(k)}^{+}$for $P_{I_{2}(k)}$ and show that a natural homomorphism from $P_{I_{2}(k)}^{+}$to $P_{I_{2}(k)}$ is injective (see Proposition 2.7). This is by no means obvious (cf. [G], [B-Sai], [D], [E] and [Mic]). Here, we prove it using an argument similar to that given in $[\mathrm{G}]$. (In [F-S 2], an alternative proof is given.) The injectivity of the map from $P_{I_{2}(k)}^{+}$to $P_{I_{2}(k)}$ implies many fundamental results. The most important of these are presented in Proposition 2.9 and Lemma 3.1. 


\section{§2. Pure Artin groups of dihedral type}

Let $k$ be an integer greater than 2, and let $G_{I_{2}(k)}$ be the Artin group of dihedral type $I_{2}(k)$, which is defined by

$$
G_{I_{2}(k)}:=\left\langle\sigma_{1}, \sigma_{2} \mid\left\langle\sigma_{1} \sigma_{2}\right\rangle^{k}=\left\langle\sigma_{2} \sigma_{1}\right\rangle^{k}\right\rangle
$$

where

$$
\left\langle\sigma_{i} \sigma_{j}\right\rangle^{k}:=\underbrace{\sigma_{i} \sigma_{j} \sigma_{i} \sigma_{j} \sigma_{i} \cdots}_{k \text { letters }} .
$$

The Coxeter group of dihedral type is a group presented by

$$
\bar{G}_{I_{2}(k)}:=\left\langle\sigma_{1}, \sigma_{2} \mid\left\langle\sigma_{1} \sigma_{2}\right\rangle^{k}=\left\langle\sigma_{2} \sigma_{1}\right\rangle^{k}, \sigma_{1}^{2}=\sigma_{2}^{2}=1\right\rangle .
$$

The group $\bar{G}_{I_{2}(k)}$ is the dihedral group of order $2 k$. Let $\sigma:=\sigma_{1} \sigma_{2}$ and $\tau:=\sigma_{2}$. Then we have the usual presentation of the dihedral group:

$$
\left\langle\sigma, \tau \mid \sigma^{k}=\tau^{2}=(\sigma \tau)^{2}=1\right\rangle
$$

Next, note that there is a natural surjective homomorphism

$$
p: G_{I_{2}(k)} \rightarrow \bar{G}_{I_{2}(k)} \text {. }
$$

Let us call the kernel of $p$ the pure Artin group of dihedral type and write it $P_{I_{2}(k)}$. Then, using the Reidemeister-Schreier method, we obtain the following presentation of the pure Artin group of dihedral type (cf. [F-S 2]):

$$
\begin{aligned}
& P_{I_{2}(k)}= \\
& \quad\left\langle a_{1}, \ldots, a_{k} \mid a_{1} \cdots a_{k}=a_{2} \cdots a_{k} a_{1}=a_{3} \cdots a_{k} a_{1} a_{2}=\cdots=a_{k} a_{1} \cdots a_{k-1}\right\rangle .
\end{aligned}
$$

Example 2.1 (Case $k=3$ ). The pure braid group of three strands is a geometric realization of the pure Artin group

$$
P_{I_{2}(3)}=\left\langle a_{1}, a_{2}, a_{3} \mid a_{1} a_{2} a_{3}=a_{2} a_{3} a_{1}=a_{3} a_{1} a_{2}\right\rangle .
$$

The generators $a_{1}, a_{2}$ and $a_{3}$ are themselves braids and are given in terms of the standard Artin generators of the braid group of three strands, $\sigma_{1}$ and $\sigma_{2}$, as follows: $a_{1}=\sigma_{1}^{2} \sigma_{2}^{2}, a_{2}=\sigma_{1} \sigma_{2}^{2} \sigma_{1}$ and $a_{3}=\sigma_{1}^{-2}$. Now, let $A_{12}:=a_{3}^{-1}=\sigma_{1}^{2}$, $A_{13}:=a_{3} a_{2}=\sigma_{1}^{-1} \sigma_{2}^{2} \sigma_{1}=\sigma_{2} \sigma_{1}^{2} \sigma_{2}^{-1}$ and $A_{23}:=a_{3} a_{1}=\sigma_{2}^{2}$. Then we obtain the same presentation of $P_{I_{2}(k)}$ :

$$
P_{I_{2}(3)}=\left\langle A_{12}, A_{13}, A_{23} \mid A_{12} A_{13} A_{23}=A_{13} A_{23} A_{12}=A_{23} A_{12} A_{13}\right\rangle .
$$

Here, note that $\left\{A_{12}, A_{13}, A_{23}\right\}$ is the standard generating set of the pure braid group of three strands (cf. $[\mathrm{Bi}])$. 
The group $P_{I_{2}(k)}$ can also be realized geometrically as complex line arrangements in the complex 2-dimensional space $\mathbb{C}^{2}$. More precisely, the fundamental group of the complement of $k$ complex lines in $\mathbb{C}^{2}$ that intersect each other at the origin has the presentation (1) with an appropriate base point (cf. [R]).

Now, we define the sets

$$
A^{+}:=\left\{a_{1}, \ldots, a_{k}\right\}, \quad A^{-}:=\left\{a_{1}^{-1}, \ldots, a_{k}^{-1}\right\}, \quad A:=A^{+} \cup A^{-} .
$$

Then, let $A^{*},\left(A^{+}\right)^{*}$ and $\left(A^{-}\right)^{*}$ be the free monoids generated by $A, A^{+}$and $A^{-}$, respectively. We refer to $A$ as an alphabet, its elements as letters, and the elements of $A^{*}$ as words. The elements of $A^{+}$and $A^{-}$are referred to as positive letters and negative letters, respectively, while the elements of $\left(A^{+}\right)^{*}$ and $\left(A^{-}\right)^{*}$ are referred to as positive words and negative words, respectively. The length of a word $w$ is the number of letters it contains, which is denoted by $|w|$. The length of the null word, $\varepsilon$, is zero. The null word is the identity of each monoid.

We write the canonical monoid homomorphism as $\pi: A^{*} \rightarrow P_{I_{2}(k)}$. If $u$ and $v$ are words, then $u=v$ means that $\pi(u)=\pi(v)$ and $u \equiv v$ means that $u$ and $v$ are identical letter by letter. A word $w \in \pi^{-1}(g)$ is called a word of $g$. The length of a group element $g$ is regarded as the quantity

$$
\|g\|=\min \left\{l \mid g=\pi\left(s_{1} \cdots s_{l}\right), s_{i} \in A\right\} .
$$

A word $w \in A^{*}$ is geodesic if $|w|=\|\pi(w)\|$. A word $w_{1} \cdots w_{m} \in A^{*}$ is called a reduced word if $w_{i} \neq w_{i+1}^{-1}$ for all $i \in\{1, \ldots, m-1\}$. A geodesic representative is a reduced word.

Now, for each $q \in \mathbb{N} \cup\{0\}$, we make the definition

$$
\alpha_{q}:=\sharp\left\{g \in P_{I_{2}(k)} \mid\|g\|=q\right\} .
$$

The spherical growth series of $P_{I_{2}(k)}$ with respect to $A$ is the formal power series

$$
\mathcal{S}_{P_{I_{2}(k)}}(t):=\sum_{q=0}^{\infty} \alpha_{q} t^{q} .
$$

It is well known that the radius of convergence of the spherical growth series of any finitely generated group is positive. Thus, $\mathcal{S}_{P_{I_{2}(k)}}(t)$ is a holomorphic function near the origin, 0 .

As a group, $P_{I_{2}(k)}$ is isomorphic to $\mathbb{Z} \times F_{k-1}$, where $F_{k-1}$ is the free group of rank $k-1$ (cf. [F-S 2]). Hence, the group structure of $P_{I_{2}(k)}$ is quite simple. In this paper, however, we wish to elucidate the combinatorial group structure of $P_{I_{2}(k)}$ with respect to the presentation (1). In particular, we investigate the growth series of the group $P_{I_{2}(k)}$ with respect to the generating set 
$A=\left\{a_{1}, \ldots, a_{k}, a_{1}^{-1}, \ldots, a_{k}^{-1}\right\}$. Note that the rationality of the spherical growth series depends on the generating set for some groups (cf. [St]). We will see in this paper that the spherical growth series $\mathcal{S}_{P_{2(k)}}(t)$ of $P_{I_{2}(k)}$ with respect to $A$ is rational.

The pure Artin monoid of dihedral type is a monoid presented by

$$
P_{I_{2}(k)}^{+}:=\left\langle a_{1}, \ldots, a_{k} \mid a_{1} \cdots a_{k}=a_{2} \cdots a_{k} a_{1}=\cdots=a_{k} a_{1} \cdots a_{k-1}\right\rangle^{+},
$$

where the right-hand side is the quotient of the free monoid $\left(A^{+}\right)^{*}$ by an equivalence relation on $\left(A^{+}\right)^{*}$ defined as follows: (i) two positive words $\omega, \omega^{\prime} \in\left(A^{+}\right)^{*}$ are elementarily equivalent if there are positive words $u, v \in\left(A^{+}\right)^{*}$ and indices $i, j \in$ $\{1, \ldots, k\}$ such that $\omega \equiv u\left(a_{i} \cdots a_{k} a_{1} \cdots a_{i-1}\right) v$ and $\omega^{\prime} \equiv u\left(a_{j} \cdots a_{k} a_{1} \cdots a_{j-1}\right) v$; (ii) two positive words $\omega, \omega^{\prime} \in\left(A^{+}\right)^{*}$ are equivalent if there is a sequence $\omega_{0} \equiv$ $\omega, \omega_{1}, \ldots, \omega_{l} \equiv \omega^{\prime}$ for some $l \in \mathbb{N} \cup\{0\}$ such that $\omega_{s}$ is elementarily equivalent to $\omega_{s+1}$ for $s=0, \ldots, l-1$. We say that $\omega^{\prime}$ is obtained from $\omega$ by a positive transformation of chain length $l$. If two positive words $\omega$ and $\omega^{\prime}$ belong to the same equivalence class, we say that they are positively equal and write $\omega \doteq \omega^{\prime}$. If $\omega \equiv \omega^{\prime}$, then $\omega \doteq \omega^{\prime}$ by definition. It is also obvious that if $\omega \doteq \omega^{\prime}$, then $\omega=\omega^{\prime}$ and $|\omega|=\left|\omega^{\prime}\right|$.

There is a natural homomorphism $P_{I_{2}(k)}^{+} \rightarrow P_{I_{2}(k)}$. Below, we show that this homomorphism is injective by following the argument given in $[\mathrm{G}]$. (For another proof, see [F-S 2].)

We first show the following:

Lemma 2.2. Let $X, Y \in\left(A^{+}\right)^{*}$. Assume that $a_{i} X \doteq a_{j} Y$ for $i, j \in\{1, \ldots, k\}$. Then the following hold:

(i) If $j=i$, then $X \doteq Y$.

(ii) If $j \neq i$, then there exists a positive word $Z \in\left(A^{+}\right)^{*}$ such that

$$
X \doteq\left(a_{i+1} \cdots a_{k} \cdot a_{1} \cdots a_{i-1}\right) Z \quad \text { and } \quad Y \doteq\left(a_{j+1} \cdots a_{k} \cdot a_{1} \cdots a_{j-1}\right) Z
$$

Proof. We refer to the assertion of this lemma in the case of words $X$ and $Y$ of length $r$ as $\mathbf{L}_{r}$. For $r=0,1, \ldots, k-1$, this assertion takes simpler forms that are readily proven. These are given below.

$\mathbf{L}_{r}(0 \leq r \leq k-2)$ : When $X$ and $Y$ are positive words of length $r$, the following hold:

1. If $a_{i} X \doteq a_{i} Y$, then $X \equiv Y$.

2. If $j \neq i$ and $a_{i} X \doteq a_{j} Y$, then $a_{i} X$ cannot be positively equal to $a_{j} Y$. 
$\mathbf{L}_{k-1}$ : When $X$ and $Y$ are positive words of length $k-1$, the following hold:

1. If $a_{i} X \doteq a_{i} Y$, then $X \equiv Y$.

2. If $j \neq i$ and $a_{i} X \doteq a_{j} Y$, then

$$
X \equiv a_{i+1} \cdots a_{k} \cdot a_{1} \cdots a_{i-1} \quad \text { and } \quad Y \equiv a_{j+1} \cdots a_{k} \cdot a_{1} \cdots a_{j-1} .
$$

The proof for the general $r \geq k$ case is obtained by applying induction twice, first with regard to the length of $X$, and then with regard to the chain length of the positive transformation. As the induction hypothesis, we assume

( $\alpha$ ) $\mathbf{L}_{r}$ holds for $0 \leq r \leq R$ for all positive transformations of all chain lengths $l$, and

( $\beta) \mathbf{L}_{R+1}$ holds for positive transformations of all chain lengths $l \leq L$.

Now, let $X$ and $Y$ be positive words of length $R+1$ and suppose that $a_{i} X \doteq a_{j} Y$ holds through a positive transformation of chain length $L+1$. Let the successive positive words of the transformation be

$$
W_{1} \equiv a_{i} X \rightarrow W_{2} \rightarrow W_{3} \rightarrow \cdots \rightarrow W_{L+2} \equiv a_{j} Y .
$$

Next, arbitrarily choose any intermediate word $W_{n} \equiv a_{m} W$ from the sequence, where $n \neq 1, L+2$ and $|W|=R+1$. Then we have

$$
a_{i} X \doteq a_{m} W \doteq a_{j} Y \text {. }
$$

The transformations $a_{i} X \rightarrow a_{m} W$ and $a_{m} W \rightarrow a_{j} Y$ are each of chain length $l \leq L$. Thus, we can apply $(\beta)$ to them. We now show that $\mathbf{L}_{R+1}$ holds for $X$ and $Y$. This is done by considering five cases regarding $i, m$ and $j$ separately.

Case 1: $j=i$ and $m=i$. From (2), we have

$$
a_{i} X \doteq a_{i} W \doteq a_{i} Y
$$

Then, from $(\beta)$, it follows that $X \doteq W$ and $W \doteq Y$. Thus, $X \doteq Y$.

Case 2: $j=i$ and $m \neq i$. From (2), we have

$$
a_{i} X \doteq a_{m} W \doteq a_{i} Y \text {. }
$$

From $(\beta)$, it follows that there exist positive words $U$ and $V$ such that

$$
\begin{aligned}
X \doteq\left(a_{i+1} \cdots a_{k} \cdot a_{1} \cdots a_{i-1}\right) U, & W \doteq\left(a_{m+1} \cdots a_{k} \cdot a_{1} \cdots a_{m-1}\right) U, \\
W & \doteq\left(a_{m+1} \cdots a_{k} \cdot a_{1} \cdots a_{m-1}\right) V, \quad Y \doteq\left(a_{i+1} \cdots a_{k} \cdot a_{1} \cdots a_{i-1}\right) V .
\end{aligned}
$$

Thus, we have $\left(a_{m+1} \cdots a_{k} \cdot a_{1} \cdots a_{m-1}\right) U \doteq\left(a_{m+1} \cdots a_{k} \cdot a_{1} \cdots a_{m-1}\right) V$. Here, note that $\left|\left(a_{m+2} \cdots a_{k} \cdot a_{1} \cdots a_{m-1}\right) U\right|=\left|\left(a_{m+2} \cdots a_{k} \cdot a_{1} \cdots a_{m-1}\right) V\right|=R$. Then, 
by $(\alpha)$, we obtain $\left(a_{m+2} \cdots a_{k} \cdot a_{1} \cdots a_{m-1}\right) U \doteq\left(a_{m+2} \cdots a_{k} \cdot a_{1} \cdots a_{m-1}\right) V$. Repeating this procedure, we finally obtain $U \doteq V$. Hence,

$$
X \doteq\left(a_{i+1} \cdots a_{k} \cdot a_{1} \cdots a_{i-1}\right) U \doteq\left(a_{i+1} \cdots a_{k} \cdot a_{1} \cdots a_{i-1}\right) V \doteq Y .
$$

Case 3: $j \neq i$ and $m=i$. From (2), we have

$$
a_{i} X \doteq a_{i} W \doteq a_{j} Y
$$

From $(\beta)$, it follows that $X \doteq W$ and there exists a positive word $V$ such that $W \doteq\left(a_{i+1} \cdots a_{k} \cdot a_{1} \cdots a_{i-1}\right) V$ and $Y \doteq\left(a_{j+1} \cdots a_{k} \cdot a_{1} \cdots a_{j-1}\right) V$. Thus, we have $X \doteq\left(a_{i+1} \cdots a_{k} \cdot a_{1} \cdots a_{i-1}\right) V$ and $Y \doteq\left(a_{j+1} \cdots a_{k} \cdot a_{1} \cdots a_{j-1}\right) V$.

Case 4: $j \neq i$ and $m=j$. From (2), we have

$$
a_{i} X \doteq a_{j} W \doteq a_{j} Y
$$

From $(\beta)$, it follows that $W \doteq Y$, and there exists a positive word $U$ such that $X \doteq\left(a_{i+1} \cdots a_{k} \cdot a_{1} \cdots a_{i-1}\right) U$ and $W \doteq\left(a_{j+1} \cdots a_{k} \cdot a_{1} \cdots a_{j-1}\right) U$. Thus, we have $X \doteq\left(a_{i+1} \cdots a_{k} \cdot a_{1} \cdots a_{i-1}\right) U$ and $Y \doteq\left(a_{j+1} \cdots a_{k} \cdot a_{1} \cdots a_{j-1}\right) U$.

Case 5: $j \neq i$ and $m \neq i, j$. Here, we have the equality (2). From ( $\beta$ ), it follows that there exist positive words $U$ and $V$ such that

$$
\begin{aligned}
X \doteq\left(a_{i+1} \cdots a_{k} \cdot a_{1} \cdots a_{i-1}\right) U, & W \doteq\left(a_{m+1} \cdots a_{k} \cdot a_{1} \cdots a_{m-1}\right) U \\
W & \doteq\left(a_{m+1} \cdots a_{k} \cdot a_{1} \cdots a_{m-1}\right) V, \quad Y \doteq\left(a_{j+1} \cdots a_{k} \cdot a_{1} \cdots a_{j-1}\right) V
\end{aligned}
$$

Thus, we have $\left(a_{m+1} \cdots a_{k} \cdot a_{1} \cdots a_{m-1}\right) U \doteq\left(a_{m+1} \cdots a_{k} \cdot a_{1} \cdots a_{m-1}\right) V$. Then, applying $(\alpha)$ repeatedly, we obtain $U \doteq V$. Hence, $X \doteq\left(a_{i+1} \cdots a_{k} \cdot a_{1} \cdots a_{i-1}\right) U \doteq$ $\left(a_{i+1} \cdots a_{k} \cdot a_{1} \cdots a_{i-1}\right) V$ and $Y \doteq\left(a_{j+1} \cdots a_{k} \cdot a_{1} \cdots a_{j-1}\right) V$.

It is obvious that $\mathbf{L}_{R+1}$ is true for chain length 1 . Hence, by induction with regard to the chain length, $\mathbf{L}_{R+1}$ holds for all chain lengths. Finally, applying induction to $R$ completes the proof.

The following lemma is similar.

Lemma 2.3. Let $X, Y \in\left(A^{+}\right)^{*}$. Assume that $X a_{i} \doteq Y a_{j}$ for $i, j \in\{1, \ldots, k\}$. Then the following hold:

(i) If $j=i$, then $X \doteq Y$.

(ii) If $j \neq i$, then there exists a positive word $Z \in\left(A^{+}\right)^{*}$ such that $X \doteq Z\left(a_{i+1} \cdots a_{k}\right.$. $\left.a_{1} \cdots a_{i-1}\right)$ and $Y \doteq Z\left(a_{j+1} \cdots a_{k} \cdot a_{1} \cdots a_{j-1}\right)$. 
As an immediate consequence of Lemmas 2.2(i) and 2.3(i), we obtain

Proposition 2.4 (Cancellativity). Let $X, Y, U, V, W$ and $Z$ be positive words. Then

$$
U \doteq W, V \doteq Z, U X V \doteq W Y Z \Rightarrow X \doteq Y
$$

Next, we introduce the following positive word:

$$
\nabla:=a_{1} \cdots a_{k} \in\left(A^{+}\right)^{*}
$$

This word satisfies the relation

$$
\nabla \equiv a_{1} \cdots a_{k} \doteq a_{2} \cdots a_{k} a_{1} \doteq a_{3} \cdots a_{k} a_{1} a_{2} \doteq \cdots \doteq a_{k} a_{1} \cdots a_{k-1}
$$

From (3), we obtain

Lemma 2.5. (i) For each $a_{j} \in A^{+}$, we have $a_{j} \nabla \doteq \nabla a_{j}$.

(ii) For each $a_{j} \in A^{+}$, there exists a positive word $W \in\left(A^{+}\right)^{*}$ such that $\nabla \doteq W a_{j}$.

From Lemma 2.5 , we immediately obtain

Lemma 2.6 (Right-reversible). If $X$ and $Y$ are positive words, then there exist positive words $U$ and $V$ such that $U X \doteq V Y$.

Proof. Let $X \equiv r_{1} \cdots r_{m}$ and $Y \equiv s_{1} \cdots s_{n}$ be any positive words, where $r_{i}$ and $s_{i}$ are elements of $A^{+}$. Then, by Lemma 2.5 , we have

$$
\nabla^{n} X \doteq \nabla^{n-1} W_{1} s_{n} \doteq \nabla^{n-2} W_{2} s_{n-1} s_{n} \doteq \cdots \doteq W_{n} Y,
$$

where $W_{1}, \ldots, W_{n}$ are some positive words. The result follows by choosing $U \equiv \nabla^{n}$ and $V \equiv W_{n}$.

With the above preparation, we obtain the following:

Proposition 2.7 ([F-S 2]). The homomorphism $P_{I_{2}(k)}^{+} \rightarrow P_{I_{2}(k)}$ is injective.

Proof. By Proposition 2.4 and Lemma 2.6, the pure Artin monoid $P_{I_{2}(k)}^{+}$is cancellative and right-reversible. Hence, by Öre's theorem [O], $P_{I_{2}(k)}^{+}$can be embedded in a group, say $Q$. Let $Q^{\prime}$ be the subgroup of $Q$ generated by $a_{1}, \ldots, a_{k}$. Then $P_{I_{2}(k)}^{+}$ is embedded in $Q^{\prime}$.

Now, suppose that $X$ and $Y$ are any positive words such that $X=Y$ in the group $P_{I_{2}(k)}$. Then, because the relations of $Q^{\prime}$ include those defining $P_{I_{2}(k)}$, we have $X=Y$ in the group $Q^{\prime}$ also. This implies that $X \doteq Y$, since $P_{I_{2}(k)}^{+}$is embedded in $Q^{\prime}$. 
In this paper, we consider $P_{I_{2}(k)}^{+}$to be a subset of $P_{I_{2}(k)}$, identifying the null word $\varepsilon$ with the identity of $P_{I_{2}(k)}$. Then we have the following commutative diagram:

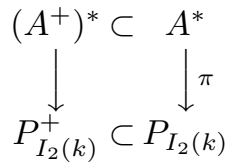

Recall that for $u, v \in A^{*}$, the expression $u=v$ means that $\pi(u)=\pi(v)$. Thus, Proposition 2.7 implies that for $u, v \in\left(A^{+}\right)^{*}$, we have $u=v \Leftrightarrow u \doteq v$, and for $u, v \in\left(A^{-}\right)^{*}$, we have $u=v \Leftrightarrow u^{-1} \doteq v^{-1}$. Note also that $\nabla$ satisfies

$$
\nabla \equiv a_{1} \cdots a_{k}=a_{2} \cdots a_{k} a_{1}=a_{3} \cdots a_{k} a_{1} a_{2}=\cdots=a_{k} a_{1} \cdots a_{k-1} .
$$

In order to elucidate the combinatorial structure of $P_{I_{2}(k)}$, let us introduce the concepts of fundamental blocks. A fundamental block is a positive word with length smaller than $k$ that appears as a subword in representatives of $\pi(\nabla)$ within $\left(A^{+}\right)^{*}$ (see the equality $\left.(4)\right)$. There are $k(k-1)$ fundamental blocks. We list them below:

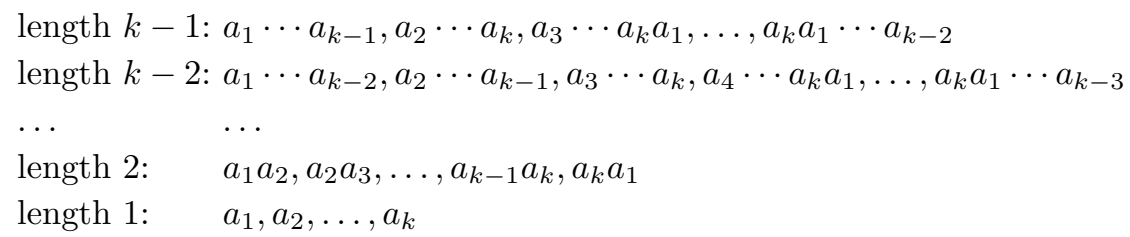

Next, we give several definitions:

$$
\begin{aligned}
& \mathrm{FB}^{+}:=\left\{\mu \in\left(A^{+}\right)^{*} \mid \mu \text { is a fundamental block }\right\} \\
& \mathrm{FB}^{-}:=\left\{\mu^{-1} \in\left(A^{-}\right)^{*} \mid \mu \in \mathrm{FB}^{+}\right\}
\end{aligned}
$$

for $I \in\{0, \ldots, k-1\}$,

$$
\mathrm{FB}_{I}^{ \pm}:=\left\{\mu \in \mathrm{FB}^{ \pm}|| \mu \mid=I\right\}, \quad \mathrm{FB}_{\leq I}^{ \pm}:=\left\{\mu \in \mathrm{FB}^{ \pm}|| \mu \mid \leq I\right\}
$$

for $\mu \equiv a_{i} \cdots a_{k} a_{1} \cdots a_{j} \in \mathrm{FB}^{+}$,

$$
\mathcal{L}(\mu):=a_{i}, \quad \mathcal{R}(\mu):=a_{j} ;
$$

for $\mu^{-1} \equiv a_{j}^{-1} \cdots a_{1}^{-1} a_{k}^{-1} \cdots a_{i}^{-1} \in \mathrm{FB}^{-}$,

$$
\mathcal{L}\left(\mu^{-1}\right):=a_{j}, \quad \mathcal{R}\left(\mu^{-1}\right):=a_{i} .
$$

For $\mu \equiv a_{i} \cdots a_{k} a_{1} \cdots a_{j} \in \mathrm{FB}^{+}$, we call $a_{j+1}$ the letter subsequent to $\mu$. When $\mu \equiv a_{i} \cdots a_{k}$, we call $a_{1}$ the letter subsequent to $\mu$. The letter subsequent to $\mu$ is denoted by $\mathcal{N}(\mu)$. For $\mu^{-1} \equiv a_{j}^{-1} \cdots a_{1}^{-1} a_{k}^{-1} \cdots a_{i}^{-1} \in \mathrm{FB}^{-}$, we call $a_{i-1}$ the letter 
subsequent to $\mu^{-1}$, which is denoted by $\mathcal{N}\left(\mu^{-1}\right)$. When $a_{i}^{-1} \equiv a_{1}^{-1}$, we call $a_{k}$ the letter subsequent to $\mu^{-1}$

Let $\Lambda$ be a subset of $\mathrm{FB}^{+} \cup \mathrm{FB}^{-} \cup\left\{\nabla, \nabla^{-1}\right\}$. Further, let $\Lambda^{*}$ be the free monoid generated by $\Lambda$. In this paper, we frequently express an element of $A^{*}$ as an element of $\Lambda^{*}$. In $\S 3$, we choose a unique geodesic representative for $g \in P_{I_{2}(k)}$ that can be expressed as an element of $\Lambda^{*}$. Then, in $\S 4$, we construct automata over $\Lambda$ that recognize such geodesic representatives.

It is easy to show that the following lemma holds.

Lemma 2.8 ([F-S 2]). (i) The element $\pi(\nabla)$ is a generator of the center of the group $P_{I_{2}(k)}$. In particular,

$$
a \nabla^{ \pm 1}=\nabla^{ \pm 1} a \quad \text { for } a \in A=A^{+} \cup A^{-} .
$$

(ii) For each $\mu \in \mathrm{FB}^{ \pm}$, there exists a unique element $\bar{\mu} \in \mathrm{FB}^{\mp}$ such that $\mu \nabla^{\mp 1}$ $=\bar{\mu}$. It satisfies $\mathcal{R}(\bar{\mu})=\mathcal{N}(\mu), \mathcal{N}(\bar{\mu})=\mathcal{R}(\mu)$ and $\mathcal{L}(\bar{\mu})=\mathcal{N}\left(\mu^{-1}\right)$, and its length satisfies $|\bar{\mu}|=k-|\mu|$.

(iii) For each $\xi \in\left(A^{+}\right)^{*}$ that contains no positive word $u$ satisfying $u=\nabla$, there exists a unique element $\mu_{1} \cdots \mu_{m} \in\left(\mathrm{FB}^{+}\right)^{*}$ such that $\xi \equiv \mu_{1} \cdots \mu_{m}$, where $\mathcal{N}\left(\mu_{j}\right) \neq \mathcal{L}\left(\mu_{j+1}\right)$ for $1 \leq j \leq m-1$.

Let $g \in P_{I_{2}(k)}$. Consider an arbitrary representative $w$ of $g$ and suppose that it contains $n_{+}$instances of a positive word $u$ satisfying $u=\nabla$ and $n_{-}$instances of a negative word $v$ satisfying $v=\nabla^{-1}$. Then, by Lemma 2.8(i), we can obtain a distinct representative $w^{\prime}$ of $g$ by moving each of these instances (in arbitrary order) to the rightmost position of $w$. Write the word $w^{\prime}$ as $w^{\prime} \equiv w_{1} \cdots w_{n} \cdot \nabla^{c}$, where $c:=n_{+}-n_{-}$, and $w_{j} \in A$ for each $1 \leq j \leq n$. If there exists $j$ such that $w_{j+1}=w_{j}^{-1}$, we reduce $w_{j} \cdot w_{j+1}$ to the null word $\varepsilon$. Repeating this reduction procedure, we obtain a reduced word $w^{\prime \prime}$ satisfying $w^{\prime \prime}=w_{1} \cdots w_{n}$. This yields the representative $\xi$ given by $\xi:=w^{\prime \prime} \cdot \nabla^{c}$. We present $\xi$ as

$$
\xi \equiv x^{(1)} \cdot y^{(1)} \cdot x^{(2)} \cdot y^{(2)} \cdots x^{(l)} \cdot y^{(l)} \cdot \nabla^{c},
$$

where for any $i \in\{1, \ldots, l\}$, we have

$$
x^{(i)} \in\left(A^{+}\right)^{*}, \quad y^{(i)} \in\left(A^{-}\right)^{*} .
$$

Furthermore, by Lemma 2.8(iii), for each $i \in\{1, \ldots, l\}$, the words $x^{(i)}$ and $y^{(i)}$ are uniquely expressed as elements of $\left(\mathrm{FB}^{+}\right)^{*}$ and $\left(\mathrm{FB}^{-}\right)^{*}$, respectively, as

$$
x^{(i)} \equiv x_{1}^{(i)} \cdots x_{k_{i}}^{(i)} \in\left(\mathrm{FB}^{+}\right)^{*} \quad \text { and } \quad y^{(i)} \equiv y_{1}^{(i)} \cdots y_{K_{i}}^{(i)} \in\left(\mathrm{FB}^{-}\right)^{*}
$$


where

$$
\begin{cases}\mathcal{N}\left(x_{j}^{(i)}\right) \neq \mathcal{L}\left(x_{j+1}^{(i)}\right) & \text { for } j \in\left\{1, \ldots, k_{i}-1\right\} \\ \mathcal{N}\left(y_{j}^{(i)}\right) \neq \mathcal{L}\left(y_{j+1}^{(i)}\right) & \text { for } j \in\left\{1, \ldots, K_{i}-1\right\} \\ \mathcal{R}\left(x_{k_{i}}^{(i)}\right) \neq \mathcal{L}\left(y_{1}^{(i)}\right), & \\ \mathcal{R}\left(y_{K_{i}}^{(i)}\right) \neq \mathcal{L}\left(x_{1}^{(i+1)}\right) . & \end{cases}
$$

We note that the subword $x^{(1)} \cdot y^{(1)} \cdots x^{(l)} \cdot y^{(l)}$ is a reduced word. From $\xi$, we obtain a particular representative of $g$, as done in [G].

Proposition 2.9 (Normal form). For each $g \in P_{I_{2}(k)}$, there exist unique $\mu_{1} \cdots \mu_{m}$ $\in\left(\mathrm{FB}^{+}\right)^{*}$ and $d \in \mathbb{Z}$ such that $\nu:=\mu_{1} \cdots \mu_{m} \cdot \nabla^{d}$ is a representative of $g$, where $\mathcal{N}\left(\mu_{j}\right) \neq \mathcal{L}\left(\mu_{j+1}\right)$ for $1 \leq j \leq m-1$. We call $\nu \equiv \mu_{1} \cdots \mu_{m} \cdot \nabla^{d}$ the normal form of $g$ and $\mu_{1} \cdots \mu_{m}$ the non- $\nabla$ part of the normal form.

Proof. Consider a representative $\xi$ of $g$ taking the form in (5). Then, by Lemma 2.8(ii), for each $y_{j}^{(i)}$ in (7) there exists a fundamental block $\bar{y}_{j}^{(i)} \in \mathrm{FB}^{+}$such that $y_{j}^{(i)} \nabla=\bar{y}_{j}^{(i)}$. Therefore, $y_{j}^{(i)}=\bar{y}_{j}^{(i)} \nabla^{-1}$. From $\xi$, we thus obtain another representative of $g$ as

$$
\left(x_{1}^{(1)} \cdots x_{k_{1}}^{(1)}\right) \cdot\left(\bar{y}_{1}^{(1)} \nabla^{-1} \cdots \bar{y}_{K_{1}}^{(1)} \nabla^{-1}\right) \cdots\left(x_{1}^{(l)} \cdots x_{k_{l}}^{(l)}\right) \cdot\left(\bar{y}_{1}^{(l)} \nabla^{-1} \cdots \bar{y}_{K_{l}}^{(l)} \nabla^{-1}\right) \cdot \nabla^{c} .
$$

Then, moving every $\nabla^{-1}$ to the right, we obtain the following representative:

$$
\left(x_{1}^{(1)} \cdots x_{k_{1}}^{(1)}\right) \cdot\left(\bar{y}_{1}^{(1)} \cdots \bar{y}_{K_{1}}^{(1)}\right) \cdots\left(x_{1}^{(l)} \cdots x_{k_{l}}^{(l)}\right) \cdot\left(\bar{y}_{1}^{(l)} \cdots \bar{y}_{K_{l}}^{(l)}\right) \cdot \nabla^{\left(c-K_{1}-\cdots-K_{l}\right)} .
$$

Using (8) and Lemma 2.8(ii), we confirm that $\mathcal{N}\left(x_{j}^{(i)}\right) \neq \mathcal{L}\left(x_{j+1}^{(i)}\right), \mathcal{N}\left(\bar{y}_{j}^{(i)}\right) \neq$ $\mathcal{L}\left(\bar{y}_{j+1}^{(i)}\right), \mathcal{N}\left(x_{k_{i}}^{(i)}\right) \neq \mathcal{L}\left(\bar{y}_{1}^{(i)}\right)$ and $\mathcal{N}\left(\bar{y}_{K_{i}}^{(i)}\right) \neq \mathcal{L}\left(x_{1}^{(i+1)}\right)$. Therefore, (9) is the desired representative of $g$ with $m=\sum_{i=1}^{l}\left(k_{i}+K_{i}\right)$ and $d=c-\sum_{i=1}^{l} K_{i}$.

Finally, we demonstrate the uniqueness of the normal form. Assume that $\mu_{1} \cdots \mu_{m} \nabla^{d}=\nu_{1} \cdots \nu_{m^{\prime}} \nabla^{d^{\prime}}$ in $P_{I_{2}(k)}$. Suppose that $d^{\prime}<d$ and write $n:=d-d^{\prime}$ $(>0)$. Then $\mu_{1} \cdots \mu_{m} \nabla^{n}=\nu_{1} \cdots \nu_{m^{\prime}}$ in $P_{I_{2}(k)}$. Thus, by Proposition 2.7, we obtain $\mu_{1} \cdots \mu_{m} \nabla^{n} \doteq \nu_{1} \cdots \nu_{m^{\prime}}$. Hence, $\nu_{1} \cdots \nu_{m^{\prime}}$ contains a positive word $u$ satisfying $u \doteq \nabla$. This is impossible. We thus conclude that $d^{\prime} \geq d$, and similarly $d \geq$ $d^{\prime}$. Hence, $d=d^{\prime}$, and this implies, by Lemma 2.3 and Proposition 2.7, that $\mu_{1} \cdots \mu_{m} \doteq \nu_{1} \cdots \nu_{m^{\prime}}$. Consequently, because the word on each side of this equality has only one representative in $\left(A^{+}\right)^{*}$, we have $\mu_{1} \cdots \mu_{m} \equiv \nu_{1} \cdots \nu_{m^{\prime}}$. Finally, from Lemma 2.8(iii), we have $m=m^{\prime}$ and $\mu_{j} \equiv \nu_{j}$ for each $j$. 


\section{§3. Geodesic representatives of elements of $P_{I_{2}(k)}$}

In this section, we determine all of the geodesic representatives for each element $g$ of $P_{I_{2}(k)}$. Moreover, we show that even in the case that multiple geodesic representatives exist for a given element $g$, there is a criterion we can use in order to uniquely specify a single geodesic representative for $g$.

First, we note the following:

Lemma 3.1. (i) All positive and negative words are geodesic with respect to $A$.

(ii) Let $\xi$ be a positive word (resp. a negative word). Then there exists no word $\xi^{\prime} \notin\left(A^{+}\right)^{*}\left(\right.$ resp. $\left.\notin\left(A^{-}\right)^{*}\right)$ such that $\xi^{\prime}=\xi$ and $\left|\xi^{\prime}\right|=|\xi|$.

Proof. (i) First, note that the null word, $\varepsilon$, is geodesic. Next, suppose that there exists a positive word $w(\neq \varepsilon)$ that is not geodesic. There exists an element $w^{\prime} \in A^{*}$ such that $w=w^{\prime}$ in $P_{I_{2}(k)}$ and $|w|>\left|w^{\prime}\right|$. Because all of the words in the relations defining $P_{I_{2}(k)}^{+}$have the same length, $w^{\prime}$ contains at least one negative letter. Let $\left\{s_{1}^{-1}, \ldots, s_{n}^{-1}\right\}$ be the set of all such negative letters. Now, consider the product $\nabla^{n} \cdot w^{\prime}$. By Lemma 2.8(i), we move one of $\nabla$ to become a neighbor of $s_{1}^{-1}$. Then, using the relation (4), we can cancel the letters $s_{1}$ (in $\nabla$ ) and $s_{1}^{-1}$. Peforming this procedure $n$ times, we find that $\nabla^{n} \cdot w^{\prime}$ is equal to some positive word $w^{\prime \prime}$ in $P_{I_{2}(k)}$. Note that $\left|\nabla^{n} \cdot w^{\prime}\right|>\left|w^{\prime \prime}\right|$. It follows that $\nabla^{n} \cdot w=w^{\prime \prime}$ in $P_{I_{2}(k)}$ and $\left|\nabla^{n} \cdot w\right|>\left|w^{\prime \prime}\right|$. Thus, by Proposition 2.7, we have $\nabla^{n} \cdot w \doteq w^{\prime \prime}$. Then, since all of the words in the relations defining $P_{I_{2}(k)}^{+}$have the same length, we have $\left|\nabla^{n} \cdot w\right|=\left|w^{\prime \prime}\right|$, a contradiction. It can be proved in a similar manner that any negative word is geodesic.

(ii) Suppose that there exists $\xi^{\prime} \notin\left(A^{+}\right)^{*}$ such that $\xi^{\prime} \in \pi^{-1}(\xi)$ and $\left|\xi^{\prime}\right|=|\xi|$. Then $\xi^{\prime}$ contains at least one negative letter. Let $\left\{s_{1}^{-1}, \ldots, s_{n}^{-1}\right\}$ be the set of all such negative letters. By a similar argument to that given above, $\nabla^{n} \cdot \xi^{\prime}$ is equal to some positive word $\xi^{\prime \prime}$ in $P_{I_{2}(k)}$. Note that $\left|\nabla^{n} \cdot \xi^{\prime}\right|>\left|\xi^{\prime \prime}\right|$. It follows that $\nabla^{n} \cdot \xi=\xi^{\prime \prime}$ and $\left|\nabla^{n} \cdot \xi\right|>\left|\xi^{\prime \prime}\right|$. Thus, by Proposition 2.7 , we have $\nabla^{n} \cdot \xi \doteq \xi^{\prime \prime}$, and this implies that $\left|\nabla^{n} \cdot \xi\right|=\left|\xi^{\prime \prime}\right|$, a contradiction.

Next, we present a necessary condition for a word of $A^{*}$ to be geodesic.

Lemma 3.2. Let $\gamma$ be a geodesic word with respect to A. Then the following hold:

(i) If $\gamma$ contains at least one positive word $u$ satisfying $u=\nabla$, then $\gamma \in\left(A^{+}\right)^{*}$, and moreover $\gamma=x^{(1)} \cdot \nabla^{c}$, where $c>0$ and $x^{(1)} \in\left(A^{+}\right)^{*}$ satisfies $(7)$ and $(8)$.

(ii) If $\gamma$ contains at least one negative word $v$ satisfying $v=\nabla^{-1}$, then $\gamma \in\left(A^{-}\right)^{*}$, and moreover $\gamma=y^{(1)} \cdot \nabla^{c}$, where $c<0$ and $y^{(1)} \in\left(A^{-}\right)^{*}$ satisfies (7) and (8). 
(iii) If $\gamma$ contains no positive word $u$ satisfying $u=\nabla$ and no negative word $v$ satisfying $v=\nabla^{-1}$, then $\gamma \equiv x^{(1)} \cdot y^{(1)} \cdot x^{(2)} \cdot y^{(2)} \cdots x^{(l)} \cdot y^{(l)}$, where $x^{(i)} \in\left(A^{+}\right)^{*}$ and $y^{(i)} \in\left(A^{-}\right)^{*}$ satisfy $(7)$ and $(8)$.

Proof. (i) Suppose that $\gamma$ contains a negative letter $a_{i}^{-1}$. Then, by Lemma 2.8(i), we obtain a representative $\gamma^{\prime}$ by moving the word $u(=\nabla)$ so that it is a neighbor of $a_{i}^{-1}$. Then, using the relation (4), we can cancel the letters $a_{i}$ (in $u$ ) and $a_{i}^{-1}$. Hence, we obtain another representative $\gamma^{\prime \prime}$ which satisfies $\left|\gamma^{\prime \prime}\right|<\left|\gamma^{\prime}\right|(=|\gamma|)$. However, because $\gamma$ is geodesic, this is a contradiction. Therefore, $\gamma$ is a positive word. Finally, from Lemma 2.8(iii), the second assertion follows immediately.

(ii) The assertion in this case can be demonstrated similarly.

(iii) Since any geodesic is a reduced word, by Lemma 2.8(iii), $\gamma$ can be decomposed as in (5), with the conditions (6)-(8) and $c=0$.

Now, we consider the following three types of words.

Type 1: A positive word $\xi$ that satisfies

$$
\xi=x^{(1)} \cdot \nabla^{c},
$$

where $c>0$ and $x^{(1)} \in\left(A^{+}\right)^{*}$ satisfies (7) and (8). Here, we define

$$
\operatorname{Pos}(\xi):=k, \quad \operatorname{Neg}(\xi):=0 .
$$

Type 2: A negative word $\xi$ that satisfies

$$
\xi=y^{(1)} \cdot \nabla^{c},
$$

where $c<0$ and $y^{(1)} \in\left(A^{-}\right)^{*}$ satisfies (7) and (8). Here, we define

$$
\operatorname{Pos}(\xi):=0, \quad \operatorname{Neg}(\xi):=k .
$$

Type 3: A word $\xi$ that is presented as

$$
\xi \equiv x^{(1)} \cdot y^{(1)} \cdot x^{(2)} \cdot y^{(2)} \cdots x^{(l)} \cdot y^{(l)},
$$

where $x^{(i)} \in\left(A^{+}\right)^{*}$ and $y^{(i)} \in\left(A^{-}\right)^{*}$ satisfy (7) and (8). Here, we define

$$
\begin{aligned}
& \operatorname{Pos}(\xi):=\max \left\{\left|x_{j}^{(i)}\right| \mid 1 \leq i \leq l, 1 \leq j \leq k_{i}\right\}, \\
& \operatorname{Neg}(\xi):=\max \left\{\left|y_{j}^{(i)}\right| \mid 1 \leq i \leq l, 1 \leq j \leq K_{i}\right\} .
\end{aligned}
$$

These quantities satisfy the relations $0 \leq \operatorname{Pos}(\xi) \leq k-1$ and $0 \leq \operatorname{Neg}(\xi) \leq k-1$.

Next, for $I \in\{1,2,3\}$, we define

$$
\mathrm{WT}_{I}:=\left\{\xi \in A^{*} \mid \xi \text { is a word of Type } I\right\},
$$

and

$$
\mathrm{WT}:=\mathrm{WT}_{1} \cup \mathrm{WT}_{2} \cup \mathrm{WT}_{3} .
$$


Now, note that any $g \in P_{I_{2}(k)}$ has a geodesic representative. Thus, by Lemma 3.2 , any $g \in P_{I_{2}(k)}$ has a representative $\xi \in \mathrm{WT}$. Note that a word in WT is also an element of the free monoid $\left(\mathrm{FB}^{+} \cup \mathrm{FB}^{-} \cup\left\{\nabla, \nabla^{-1}\right\}\right)^{*}$.

As the first step in determining the geodesic representatives for each element of $P_{I_{2}(k)}$, we determine all of the geodesic representatives for an arbitrary element of $P_{I_{2}(k)}$ that has a representative of Type 1 or 2 .

Proposition 3.3. Let $g \in P_{I_{2}(k)}$. Suppose that $g$ has a representative $\xi \in \mathrm{WT}_{1}$ (resp. $\left.\xi \in \mathrm{WT}_{2}\right)$; i.e., $\xi \in\left(A^{+}\right)^{*}$ and $\xi=x^{(1)} \cdot \nabla^{c}$, where $c>0$ and $x^{(1)} \in\left(A^{+}\right)^{*}$ satisfies (7) and (8) (resp. $\xi \in\left(A^{-}\right)^{*}$ and $\xi=y^{(1)} \cdot \nabla^{c}$, where $c<0$ and $y^{(1)} \in\left(A^{-}\right)^{*}$ satisfies $(7)$ and $\left.(8)\right)$. Then $\xi$ is geodesic, and the set of all geodesic representatives of $g$ equals

$$
\begin{aligned}
\mathrm{M} \nabla_{g}^{+} & :=\left\{\gamma \in\left(A^{+}\right)^{*} \mid \gamma \doteq x^{(1)} \cdot \nabla^{c}\right\}, \\
\left(\text { resp. } \mathrm{M} \nabla_{g}^{-}\right. & \left.:=\left\{\gamma \in\left(A^{-}\right)^{*} \mid \gamma^{-1} \doteq\left(y^{(1)} \cdot \nabla^{c}\right)^{-1}\right\}\right),
\end{aligned}
$$

and $\mathrm{M} \nabla_{g}^{+}\left(\right.$resp. $\left.\mathrm{M} \nabla_{g}^{-}\right)$consists of more than one element.

Proof. We only consider the case of Type 1 words, because the proof for Type 2 can be carried out similarly. First, from Lemma 3.1(i), because $\xi$ is a positive word by assumption, $\xi$ is geodesic. Now, let $\nu$ be the normal form of $g$. Then, as in the proof of Proposition 2.9, we obtain $\nu \equiv x^{(1)} \cdot \nabla^{c} \in\left(A^{+}\right)^{*}$. Thus, by Lemma 3.1(i), $\nu$ is geodesic. Hence, for any geodesic representative $\gamma^{\prime}$, we have $\gamma^{\prime}=\nu$ and $\left|\gamma^{\prime}\right|=|\nu|$. By Lemma 3.1(ii), $\gamma^{\prime}$ is a positive word. Thus, by Proposition 2.7, we have $\gamma^{\prime} \doteq \nu$. Conversely, for any $\gamma^{\prime \prime} \in\left(A^{+}\right)^{*}$ satisfying $\gamma^{\prime \prime} \doteq \nu$, we have $\left|\gamma^{\prime \prime}\right|=|\nu|$. Hence, $\gamma^{\prime \prime}$ is geodesic. Therefore, $\mathrm{M} \nabla_{g}^{+}$is the set of all geodesic representatives of $g$. Finally, because $\nu$ contains $\nabla, \mathrm{M} \nabla_{g}^{+}$consists of more than one element.

Next, we present a necessary condition for Type 3 representatives of elements of $P_{I_{2}(k)}$ to be geodesic.

Proposition 3.4. Let $g \in P_{I_{2}(k)}$. Suppose that $g$ has a representative $\xi \in \mathrm{WT}_{3}$. If $\operatorname{Pos}(\xi)+\operatorname{Neg}(\xi)>k$, then $\xi$ is not a geodesic representative of $g$.

Proof. Set $P:=\operatorname{Pos}(\xi)$ and $N:=\operatorname{Neg}(\xi)$. By assumption, there are words $x_{j_{1}}^{\left(i_{1}\right)}$ and $y_{j_{2}}^{\left(i_{2}\right)}$ in $\xi$ such that

$$
\left|x_{j_{1}}^{\left(i_{1}\right)}\right|+\left|y_{j_{2}}^{\left(i_{2}\right)}\right|>k
$$

First, suppose that $i_{1} \leq i_{2}$ and consider the subword of $\xi$ given by

$$
\xi^{\prime}:=x_{j_{1}}^{\left(i_{1}\right)} \cdot v \cdot y_{j_{2}}^{\left(i_{2}\right)} .
$$


Then, by Lemma 2.8(ii), there exist $\bar{x}_{j_{1}}^{\left(i_{1}\right)} \in \mathrm{FB}^{-}$and $\bar{y}_{j_{2}}^{\left(i_{2}\right)} \in \mathrm{FB}^{+}$such that $\bar{x}_{j_{1}}^{\left(i_{1}\right)}=x_{j_{1}}^{\left(i_{1}\right)} \nabla^{-1}, \bar{y}_{j_{2}}^{\left(i_{2}\right)}=y_{j_{2}}^{\left(i_{2}\right)} \nabla,\left|x_{j_{1}}^{\left(i_{1}\right)}\right|+\left|\bar{x}_{j_{1}}^{\left(i_{1}\right)}\right|=k$ and $\left|y_{j_{2}}^{\left(i_{2}\right)}\right|+\left|\bar{y}_{j_{2}}^{\left(i_{2}\right)}\right|=k$. Consequently, by Lemma 2.8(i),

$$
\xi^{\prime}=x_{j_{1}}^{\left(i_{1}\right)} \cdot \nabla^{-1} \nabla \cdot v \cdot y_{j_{2}}^{\left(i_{2}\right)}=x_{j_{1}}^{\left(i_{1}\right)} \nabla^{-1} \cdot v \cdot y_{j_{2}}^{\left(i_{2}\right)} \nabla=\bar{x}_{j_{1}}^{\left(i_{1}\right)} \cdot v \cdot \bar{y}_{j_{2}}^{\left(i_{2}\right)} .
$$

Further, from (13), we have $\left|\bar{x}_{j_{1}}^{\left(i_{1}\right)}\right|+\left|\bar{y}_{j_{2}}^{\left(i_{2}\right)}\right|<k$. Thus, from (14), $\xi^{\prime}$ is not geodesic. Hence, $\xi$ is not geodesic either.

The case $i_{1}>i_{2}$ can be treated similarly, with the conclusion again that $\xi$ is not geodesic.

Next, we introduce a procedure that yields geodesic representatives. It is similar to that employed in [Be] and [M-M], and called the suitable-spread procedure. In $[M-M]$, there is an effective algorithm to determine whether or not words of the Artin group $G_{I_{2}(k)}$ of dihedral type are geodesic. The suitable-spread procedure plays an important role in that algorithm. Following [M-M], we now explain the procedure in the case of the pure Artin groups $P_{I_{2}(k)}$ of dihedral type.

We consider the normal form of an element $g \in P_{I_{2}(k)}$, as in Proposition 2.9:

$$
\nu \equiv \mu_{1} \cdots \mu_{m} \cdot \nabla^{d}
$$

The word $\nu$ is the input of this procedure.

[Case 1: $d \geq 0$ ] Here, by Lemma 3.1(i), the above normal form $\nu$ is itself geodesic. Thus, in this case, we regard $\nu$ as the output of this procedure.

[Case 2: $d<0, m \leq-d]$ First, let us rewrite (15) using $\delta:=-d>0$ :

$$
\nu \equiv \mu_{1} \cdots \mu_{m} \cdot\left(\nabla^{-1}\right)^{\delta}
$$

Then, moving one of the words $\nabla^{-1}$ so that it is the right-hand neighbor of $\mu_{j}$, we obtain another representative of $g$,

$$
\begin{aligned}
\mu_{1} \cdots \mu_{j-1} \cdot\left(\mu_{j} \nabla^{-1}\right) \cdot \mu_{j+1} & \cdots \mu_{m} \cdot\left(\nabla^{-1}\right)^{\delta-1} \\
& =\mu_{1} \cdots \mu_{j-1} \cdot \bar{\mu}_{j} \cdot \mu_{j+1} \cdots \mu_{m} \cdot\left(\nabla^{-1}\right)^{\delta-1}=: \nu^{\prime},
\end{aligned}
$$

where $\bar{\mu}_{j} \in \mathrm{FB}^{-}$is given in Proposition 2.8(ii). Note that

$$
\left|\nu^{\prime}\right|=|\nu|-2\left|\mu_{j}\right|
$$

Since $m \leq \delta$, by carrying out the above procedure $m$ times, we obtain the following representative of $g$ that consists entirely of negative letters:

$$
\bar{\mu}_{1} \cdots \bar{\mu}_{m} \cdot\left(\nabla^{-1}\right)^{\delta-m} .
$$


From Lemma 3.1(i), we know that this word is geodesic. In this case, this word is the output of the procedure.

[Case 3: $d<0, m>-d(=\delta)$ ] First, pick $\delta$ fundamental blocks $\mu_{j_{1}}, \ldots, \mu_{j_{\delta}}$ from among $\mu_{1}, \ldots, \mu_{m}$ so that $\left|\mu_{j_{1}}\right|+\cdots+\left|\mu_{j_{\delta}}\right|$ is maximal. Applying the above procedure to $\mu_{j_{1}}, \ldots, \mu_{j_{\delta}}$, we obtain the following representative that contains no $\nabla^{-1}$ :

$$
\widetilde{\nu}:=\mu_{1} \cdots \mu_{j_{1}-1} \cdot \bar{\mu}_{j_{1}} \cdot \mu_{j_{1}+1} \cdots \mu_{j_{n}-1} \cdot \bar{\mu}_{j_{n}} \cdot \mu_{j_{n}+1} \cdots \mu_{j_{\delta}-1} \cdot \bar{\mu}_{j_{\delta}} \cdot \mu_{j_{\delta}+1} \cdots \mu_{m} .
$$

Here, note that

$$
\mathcal{R}\left(\mu_{j_{n}-1}\right) \neq \mathcal{L}\left(\bar{\mu}_{j_{n}}\right), \quad \mathcal{R}\left(\bar{\mu}_{j_{n}}\right) \neq \mathcal{L}\left(\mu_{j_{n}+1}\right),
$$

and if there exist $j_{n}$ and $j_{n+1}$ such that $j_{n+1}=j_{n}+1$, then

$$
\mathcal{N}\left(\bar{\mu}_{j_{n}}\right) \neq \mathcal{L}\left(\bar{\mu}_{j_{n+1}}\right) \text {. }
$$

The word $\widetilde{\nu}$ is the output of the procedure. In the present case, from (16), we obtain

$$
|\widetilde{\nu}|=|\nu|-\sum_{n=1}^{\delta} 2\left|\mu_{j_{n}}\right| .
$$

Note that the choice of $\mu_{j_{1}}, \ldots, \mu_{j_{\delta}}$ maximizing $\left|\mu_{j_{1}}\right|+\cdots+\left|\mu_{j_{\delta}}\right|$ is not necessarily unique, and the output $\widetilde{\nu}$ depends on this choice. However, $\operatorname{Pos}(\widetilde{\nu})$ and $\operatorname{Neg}(\widetilde{\nu})$ do not depend on the choice. We define the set of all such choices as follows:

$$
\mathrm{CFB}_{g}:=\left\{\begin{array}{l|l}
\left(j_{1}, \ldots, j_{\delta}\right) & \begin{array}{l}
\nu \equiv \mu_{1} \cdots \mu_{m} \cdot \nabla^{-\delta}: \text { normal form of } g(m>\delta>0), \\
1 \leq j_{1}<\cdots<j_{\delta} \leq m, \\
\sum_{n=1}^{\delta}\left|j_{n}\right|: \text { maximal }
\end{array}
\end{array}\right\} .
$$

Let us call the above procedure the suitable-spread procedure, following Mairesse and Mathéus. Note that the output of this procedure is also a representative of $g$. We denote by $\mathrm{SS}_{g}$ the set consisting of all outputs of the suitable-spread procedure applied to the normal form of $g, \nu$. In Cases 1 and 2 , we have $\# \mathrm{SS}_{g}=1$. In Case 3, $\mathrm{SS}_{g}$ is bijective with $\mathrm{CFB}_{g}$. Now, it is important to note that in Case 3, any element of $\mathrm{SS}_{g}$ is also in the free monoid $\left(\mathrm{FB}_{\leq \operatorname{Pos}(\widetilde{\nu})}^{+} \cup \mathrm{FB}_{\leq \mathrm{Neg}(\widetilde{\nu})}^{-}\right)^{*}$. Then the following provides the final piece for this procedure.

Proposition 3.5. Let $g \in P_{I_{2}(k)}$ and $\nu \equiv \mu_{1} \cdots \mu_{m} \cdot \nabla^{d}$ be the normal form of $g$, as given in Proposition 2.9. Then, applying the suitable-spread procedure to $\nu$, we obtain a geodesic representative of $g$.

Proof. In the cases $d \geq 0$ and $d<0, m \leq-d$, it is already known that the output is geodesic. Hence, it remains to consider the case $d<0, m>-d$. We write 
$\delta:=-d(>0)$. First, note that there exists at least one geodesic representative of $g$. Choose one of them, say $\gamma$. Because $\gamma$ is geodesic, by Lemma 3.2, one of the following holds: (i) $\gamma$ is of Type 1 (i.e., there exists a geodesic representative $\widehat{\gamma}$ of $g=\pi(\gamma)$ written as $\widehat{\gamma} \equiv x_{1}^{(1)} \cdots x_{k_{1}}^{(1)} \cdot \nabla^{d}$ such that $\gamma=\widehat{\gamma}$, where the $x_{j}^{(1)}$ satisfy $(7)$ and (8), and $d>0$ ); (ii) $\gamma$ is of Type 2 (i.e., there exists a geodesic representative $\widehat{\gamma}$ of $g=\pi(\gamma)$ written as $\widehat{\gamma} \equiv y_{1}^{(1)} \cdots y_{K_{1}}^{(1)} \cdot \nabla^{d}$ such that $\gamma=\widehat{\gamma}$, where the $y_{j}^{(1)}$ satisfy (7) and (8), and $d<0$ ); (iii) $\gamma$ is of Type 3 (i.e., $\gamma$ takes the form appearing in (12), with the conditions (7) and (8)).

First, consider case (i). In this case, the normal form $\nu$ of $g=\pi(\gamma)=\pi(\widehat{\gamma})$ is identical to $\widehat{\gamma}$, i.e., $\nu \equiv \widehat{\gamma}$. This normal form does not satisfy the condition $d<0$. Thus, we need not consider case (i).

Next, consider case (ii). In this case, as in the proof of Proposition 2.9, we obtain the normal form of $g=\pi(\gamma)=\pi(\widehat{\gamma})$ as $\bar{y}_{1}^{(1)} \cdots \bar{y}_{K_{1}}^{(1)} \cdot \nabla^{d-K_{1}}$. From the uniqueness of this normal form, we have $m=K_{1}$ and $\delta=-d+K_{1}$. However, because $d<0$, this normal form does not satisfy the condition $m>\delta$. Thus, we need not consider case (ii) either.

Hence, we need only examine case (iii). If $K_{i}=0$ for all $i$, then $\gamma \equiv x^{(1)} \in$ $\left(A^{+}\right)^{*}$. This is the normal form of $\pi(\gamma)=g$ with $d=0$. However, it does not satisfy the condition $d<0$, and thus, we need not consider this case. Next, if $k_{i}=0$ for all $i$, then $\gamma \equiv y^{(1)} \in\left(A^{-}\right)^{*}$. Then, as in the proof of Proposition 2.9, we obtain the normal form of $g=\pi(\gamma)$ as $\bar{y}_{1}^{(1)} \cdots \bar{y}_{K_{1}}^{(1)} \cdot \nabla^{-K_{1}}$ with $m=K_{1}$ and $d=-K_{1}$. However, since $m=-d$, this normal form does not satisfy $m>-d$, and thus we need not consider this case either.

Hence, we assume that there exist $k_{i}$ and $K_{j}$ such that $k_{i} \neq 0$ and $K_{j} \neq 0$. Now, following the proof of Proposition 2.9, we obtain

$$
\nu \equiv\left(x_{1}^{(1)} \cdots x_{k_{1}}^{(1)}\right) \cdot\left(\bar{y}_{1}^{(1)} \cdots \bar{y}_{K_{1}}^{(1)}\right) \cdots\left(x_{1}^{(l)} \cdots x_{k_{l}}^{(l)}\right) \cdot\left(\bar{y}_{1}^{(l)} \cdots \bar{y}_{K_{l}}^{(l)}\right) \cdot \nabla^{\left(-K_{1}-\cdots-K_{l}\right)}
$$

as the normal form of $g=\pi(\gamma)$, with $m=\sum_{i=1}^{l}\left(k_{i}+K_{i}\right)$ and $\delta=\sum_{i=1}^{l} K_{i}$. Then we obtain the geodesic $\gamma$ from the normal form $\nu$ by applying the procedure $\left(\bar{y}_{j}^{(i)} \Rightarrow \bar{y}_{j}^{(i)} \cdot \nabla^{-1}=y_{j}^{(i)}\right)$ to $\bar{y}_{j}^{(i)}$ for all $i \in\{1, \ldots, l\}$ and $j \in\left\{1, \ldots, K_{i}\right\}$ as the suitable-spread procedure. It is clear that $\sum_{i=1}^{l} \sum_{j=1}^{K_{i}}\left|\bar{y}_{j}^{(i)}\right|$ is less than or equal to the maximal length of any $\delta$ fundamental blocks in the non- $\nabla$ part of $\nu$. (In fact, it is equal.) Therefore, from (17), the suitable-spread procedure induces a word of length $|\gamma|$. Hence, this word is geodesic. (Note that from the argument above, it follows that $\gamma$ is also obtained from the suitable-spread procedure applied to $\nu$.)

From the argument given in the proof of Proposition 3.5, we obtain the following: 
Proposition 3.6. Let $g \in P_{I_{2}(k)}$ and $\nu$ be the normal form of $g$. Suppose that $g$ has a geodesic representative $\gamma \in \mathrm{WT}_{3}$. Then $\gamma$ is obtained from the suitable-spread procedure applied to $\nu$.

Now, we give a sufficient condition for Type 3 representatives of elements of $P_{2(k)}$ to be geodesic.

Proposition 3.7. Let $g \in P_{I_{2}(k)}$ and $\nu$ be the normal form of $g$. Suppose that $g$ has a representative $\xi \in \mathrm{WT}_{3}$. Then the following hold:

(i) If $\operatorname{Pos}(\xi)+\operatorname{Neg}(\xi) \leq k$, then $\xi$ is geodesic, and the set of all geodesic representatives of $g$ is identical with $\mathrm{SS}_{g}$.

(ii) If $\operatorname{Pos}(\xi)+\operatorname{Neg}(\xi)=k$, then $\# \mathrm{SS}_{g} \geq 2$.

(iii) If $\operatorname{Pos}(\xi)+\operatorname{Neg}(\xi)<k$, then $\# \mathrm{SS}_{g}=1$.

Proof. Set $P:=\operatorname{Pos}(\xi)$ and $N:=\operatorname{Neg}(\xi)$. By assumption, we have $0 \leq P \leq k-1$ and $0 \leq N \leq k-1$.

As in the proof of Proposition 2.9, we obtain the normal form of $g=\pi(\xi)$ as

$$
\nu \equiv\left(x_{1}^{(1)} \cdots x_{k_{1}}^{(1)}\right) \cdot\left(\bar{y}_{1}^{(1)} \cdots \bar{y}_{K_{1}}^{(1)}\right) \cdots\left(x_{1}^{(l)} \cdots x_{k_{l}}^{(l)}\right) \cdot\left(\bar{y}_{1}^{(l)} \cdots \bar{y}_{K_{l}}^{(l)}\right) \cdot \nabla^{\left(-K_{1}-\cdots-K_{l}\right)} .
$$

By the definition of $N$, we have

$$
\left|y_{j}^{(i)}\right| \leq N \quad\left(1 \leq i \leq l, 1 \leq j \leq K_{i}\right) .
$$

Thus, with the assumption $P+N \leq k$, we obtain

$$
\left|\bar{y}_{j}^{(i)}\right|=k-\left|y_{j}^{(i)}\right| \geq k-N \geq P \quad\left(1 \leq i \leq l, 1 \leq j \leq K_{i}\right) .
$$

Further, by the definition of $P$, we have

$$
\left|x_{j}^{(i)}\right| \leq P \quad\left(1 \leq i \leq l, 1 \leq j \leq k_{i}\right) .
$$

First, consider the case $N=0$ (resp. $P=0$ ), in which we have $l=1$ and $y^{(1)} \equiv \varepsilon\left(\right.$ resp. $l=1$ and $\left.x^{(1)} \equiv \varepsilon\right)$; i.e., $\xi \equiv x^{(1)}$ (resp. $\left.\xi \equiv y^{(1)}\right)$. Then, by Lemma 3.1(i), $\xi$ is geodesic. Moreover, by Lemma 3.1(ii), any geodesic representative of $g$ is a positive word (resp. a negative word). Therefore, $\xi$ is the unique geodesic representative of $g$, since $\xi$ has only one representative in $\left(A^{+}\right)^{*}\left(\operatorname{resp} .\left(A^{-}\right)^{*}\right)$. Next, from (18), we have $\nu \equiv x^{(1)}$ (resp. $\left.\nu \equiv \bar{y}_{1}^{(1)} \cdots \bar{y}_{K_{1}}^{(1)} \cdot \nabla^{-K_{1}}\right)$. This implies that $\mathrm{SS}_{g}=\{\xi\}$ and $\# \mathrm{SS}_{g}=1$.

Next, consider the case of $P \geq 1$ and $N \geq 1$. Then $\xi \notin\left(A^{+}\right)^{*} \cup\left(A^{-}\right)^{*}$. Now, let us apply the suitable-spread procedure to the normal form $\nu$. As seen from $(20)$ and $(21)$, we can then choose all $\bar{y}_{j}^{(i)}$. Proceeding with such a choice, we obtain identically $\xi$. Thus, by Proposition $3.5, \xi$ is geodesic. Next, note that 
there exists no geodesic representative of Type 1 or Type 2. (Suppose that there exists a geodesic representative $\xi_{1} \in \mathrm{WT}_{1}$. The word $\xi_{1}$ must be positive. Then, by Lemma 3.1(ii), there exists no word $\xi_{1}^{\prime} \notin\left(A^{+}\right)^{*}$ satisfying both $\xi_{1}^{\prime}=\xi_{1}$ and $\left|\xi_{1}^{\prime}\right|=\left|\xi_{1}\right|$. This is a contradiction, because $|\xi|=\left|\xi_{1}\right|$ and $\xi \notin\left(A^{+}\right)^{*} \cup\left(A^{-}\right)^{*}$. The non-existence of geodesic representatives of Type 2 can be shown similarly.) Also recall that Proposition 3.6 asserts that all geodesic representatives of Type 3 can be obtained by applying the suitable-spread procedure to $\nu$. We thus conclude that any geodesic representative of $g$ is in $\mathrm{SS}_{g}$. Conversely, by Proposition 3.5, any element of $\mathrm{SS}_{g}$ is a geodesic representative of $g$. Therefore, the set of all geodesic representatives of $g$ is $\mathrm{SS}_{g}$.

Now, we consider the subcase $P, N \geq 1$ and $P+N<k$. Here, in place of (20), we have

$$
\left|\bar{y}_{j}^{(i)}\right|=k-\left|y_{j}^{(i)}\right| \geq k-N>P \quad\left(1 \leq i \leq l, 1 \leq j \leq K_{i}\right) .
$$

Hence, according to $(21)$ and $(22)$, we must choose all $\bar{y}_{j}^{(i)}$ in the suitable-spread procedure; i.e., we cannot choose any $x_{j}^{(i)}$. Therefore, in this case, $\xi$ is the unique geodesic representative obtained from the suitable-spread procedure. Hence, $\mathrm{SS}_{g}$ $=\{\xi\}$ and $\# \mathrm{SS}_{g}=1$.

Finally, we consider the subcase $P, N \geq 1$ and $P+N=k$. Here, from (20) and (21) it follows that there exists at least one fundamental block $x_{j}^{(i)}$ of length $P$. By choosing such fundamental blocks $x_{j}^{(i)}$ in the suitable-spread procedure, we obtain another geodesic representative. Thus, $\xi$ is not a unique geodesic representative, so $\# \mathrm{SS}_{g} \geq 2$.

One immediate consequence of Propositions 3.3 and 3.7 is the following:

Corollary 3.8. Let $g, g^{\prime} \in P_{I_{2}(k)}$, and let $\gamma$ and $\gamma^{\prime}$ be geodesic representatives of $g$ and $g^{\prime}$, respectively. If $(\operatorname{Pos}(\gamma), \operatorname{Neg}(\gamma)) \neq\left(\operatorname{Pos}\left(\gamma^{\prime}\right), \operatorname{Neg}\left(\gamma^{\prime}\right)\right)$, then $g \neq g^{\prime}$.

Proof. By Propositions 3.3 and 3.7, if $(\operatorname{Pos}(\gamma), \operatorname{Neg}(\gamma)) \neq\left(\operatorname{Pos}\left(\gamma^{\prime}\right), \operatorname{Neg}\left(\gamma^{\prime}\right)\right)$, then $\nu \neq \nu^{\prime}$, where $\nu$ and $\nu^{\prime}$ are the normal forms of $g$ and $g^{\prime}$, respectively. Because the normal form is unique for each element of $P_{I_{2}(k)}$, we obtain $g \neq g^{\prime}$.

From Corollary 3.8, for each $g \in P_{I_{2}(k)}$, we can define

$$
(\operatorname{Pos}(g), \operatorname{Neg}(g)):=(\operatorname{Pos}(\gamma), \operatorname{Neg}(\gamma))
$$

by choosing an arbitrary geodesic representative $\gamma$ of $g$. Note that, by Proposition 3.4 ,

$$
\operatorname{Pos}(g)+\operatorname{Neg}(g) \leq k
$$


Now, for any $g \in P_{I_{2}(k)}$, we wish to specify a unique geodesic representative. Thus, for $\operatorname{Pos}(g)+\operatorname{Neg}(g)=k$, we must establish a criterion for choosing among several such representatives. First, if $(\operatorname{Pos}(g), \operatorname{Neg}(g))=(k, 0)$, we choose the normal form of $g$ given in Proposition 2.9. Next, if $(\operatorname{Pos}(g), \operatorname{Neg}(g))=(0, k)$, we choose the word $y^{(1)} \cdot \nabla^{c}$ given in Proposition 3.3. Finally, if $(\operatorname{Pos}(g), \operatorname{Neg}(g)) \neq(k, 0),(0, k)$, we choose the unique geodesic representative identified by the following:

Proposition 3.9. Let $g \in P_{I_{2}(k)}$. With $(P, N):=(\operatorname{Pos}(g), \operatorname{Neg}(g))$, suppose that $P+N=k$ and $(P, N) \notin\{(k, 0),(0, k)\}$. Then there exists a unique geodesic representative $\eta \in \mathrm{WT}_{3}$ of $g$ such that all elements of $\mathrm{FB}_{N}^{-}$appear before all elements of $\mathrm{FB}_{P}^{+}$. In precise terms, this assertion is as follows:

$$
\begin{aligned}
& \text { there exist } \lambda \in \mathrm{FB}_{N}^{-}, \mu \in \mathrm{FB}_{P}^{+} \text {, and } \\
& \qquad\left\{\begin{array}{l}
\xi^{(1)} \equiv \xi_{1}^{(1)} \cdots \xi_{m_{1}}^{(1)} \in\left(\mathrm{FB}_{\leq P-1}^{+} \cup \mathrm{FB}_{\leq N-1}^{-}\right)^{*}, \\
\xi^{(2)} \equiv \xi_{1}^{(2)} \cdots \xi_{m_{2}}^{(2)} \in\left(\mathrm{FB}_{\leq P-1}^{+} \cup \mathrm{FB}_{\leq N}^{-}\right)^{*}, \\
\xi^{(3)} \equiv \xi_{1}^{(3)} \cdots \xi_{m_{3}}^{(3)} \in\left(\mathrm{FB}_{\leq P}^{+} \cup \mathrm{FB}_{\leq N-1}^{-}\right)^{*},
\end{array}\right.
\end{aligned}
$$

such that

$$
\eta \equiv \xi^{(1)} \cdot \lambda \cdot \xi^{(2)} \cdot \mu \cdot \xi^{(3)} \in \pi^{-1}(g)
$$

is geodesic, where for each $i \in\{1,2,3\}$, we have

$$
\left\{\begin{array}{l}
\xi_{j}^{(i)}, \xi_{j+1}^{(i)} \in \mathrm{FB}^{ \pm} \Rightarrow \mathcal{N}\left(\xi_{j}^{(i)}\right) \neq \mathcal{L}\left(\xi_{j+1}^{(i)}\right), \\
\xi_{j}^{(i)} \in \mathrm{FB}^{ \pm}, \xi_{j+1}^{(i)} \in \mathrm{FB}^{\mp} \Rightarrow \mathcal{R}\left(\xi_{j}^{(i)}\right) \neq \mathcal{L}\left(\xi_{j+1}^{(i)}\right),
\end{array} \quad\left(j \in\left\{1, \ldots, m_{i}-1\right\}\right),\right.
$$

and

$$
\left\{\begin{array}{l}
\xi_{m_{1}}^{(1)} \in \mathrm{FB}^{+}\left(\text {resp. } \mathrm{FB}^{-}\right) \Rightarrow \mathcal{R}\left(\xi_{m_{1}}^{(1)}\right) \neq \mathcal{L}(\lambda)\left(\text { resp. } \mathcal{N}\left(\xi_{m_{1}}^{(1)}\right) \neq \mathcal{L}(\lambda)\right), \\
\xi_{1}^{(2)} \in \mathrm{FB}^{+}\left(\text {resp. } \mathrm{FB}^{-}\right) \Rightarrow \mathcal{R}(\lambda) \neq \mathcal{L}\left(\xi_{1}^{(2)}\right)\left(\text { resp. } \mathcal{N}(\lambda) \neq \mathcal{L}\left(\xi_{1}^{(2)}\right)\right), \\
\xi_{m_{2}}^{(2)} \in \mathrm{FB}^{+}\left(\text {resp. } \mathrm{FB}^{-}\right) \Rightarrow \mathcal{N}\left(\xi_{m_{2}}^{(2)}\right) \neq \mathcal{L}(\mu)\left(\text { resp. } \mathcal{R}\left(\xi_{m_{2}}^{(2)} \neq \mathcal{L}(\mu)\right),\right. \\
\xi_{1}^{(3)} \in \mathrm{FB}^{+}\left(\text {resp. } \mathrm{FB}^{-}\right) \Rightarrow \mathcal{N}(\mu) \neq \mathcal{L}\left(\xi_{1}^{(3)}\right)\left(\text { resp. } \mathcal{R}(\mu) \neq \mathcal{L}\left(\xi_{1}^{(3)}\right)\right) .
\end{array}\right.
$$

Proof. (Refer to the proof of Proposition 3.7.) The word $\xi$ and the normal form $\nu$ of $g=\pi(\xi)$ have the forms (12) and (18), respectively. Rewrite (18) as

$$
\nu \equiv \mu_{1} \cdots \mu_{m} \cdot \nabla^{-\delta},
$$

where $m:=\sum_{i=1}^{l}\left(k_{i}+K_{i}\right)$ and $\delta:=\sum_{i=1}^{l} K_{i}$. Then, by applying the suitablespread procedure to $\nu$, we obtain a geodesic representative of $g$. In this procedure, we choose $\delta$ elements from the non- $\nabla$ part of $\nu$. Here, employing the assumptions $P+N=k$ and $(P, N) \notin\{(k, 0),(0, k)\}$, the procedure is performed in accordance with the following rules. 
(i) All the elements appearing in the non- $\nabla$ part that belong to $\mathrm{FB}_{I}^{+}(I \geq P+1)$ are chosen.

(ii) There are at least two elements in the non- $\nabla$ part that belong to $\mathrm{FB}_{P}^{+}$, say $\mu_{j_{1}}, \ldots \mu_{j_{n}}$, where $j_{1}<\cdots<j_{n}$. At least one and fewer than $n$ elements from among $\mu_{j_{1}}, \ldots, \mu_{j_{n}}$ are chosen.

(iii) When choosing the elements belonging to $\mathrm{FB}_{P}^{+}$, we always choose the leftmost such element. Let us refer to the elements chosen through this procedure as $\mu_{j_{1}}, \ldots, \mu_{j_{r}}$, where $1 \leq r<n$.

In this way, we obtain a geodesic representative $\eta$ containing the elements $\bar{\mu}_{j_{1}}, \ldots, \bar{\mu}_{j_{r}} \in \mathrm{FB}_{N}^{-}$and $\mu_{j_{r+1}}, \ldots, \mu_{j_{n}} \in \mathrm{FB}_{P}^{+}$. Then, choosing $\lambda$ (resp. $\mu$ ) to be the leftmost element, $\bar{\mu}_{j_{1}}$ (resp. $\mu_{j_{r+1}}$ ), we obtain the desired geodesic representative.

Example 3.10. Let $k=3$. In this example, we consider an element $g \in P_{I_{2}(3)}$ whose normal form $\nu$ is given by

$$
\begin{aligned}
\nu & \equiv a_{1} a_{2} \cdot a_{2} \cdot a_{2} a_{3} \cdot a_{3} \cdot a_{2} \cdot a_{2} a_{3} \cdot a_{2} \cdot \nabla^{-4} \\
& \equiv \mu_{1} \cdot \mu_{2} \cdot \mu_{3} \cdot \mu_{4} \cdot \mu_{5} \cdot \mu_{6} \cdot \mu_{7} \cdot \nabla^{-4} .
\end{aligned}
$$

Then, applying the suitable-spread procedure, we have

$$
\begin{aligned}
\nu= & \left(\mu_{1} \nabla^{-1}\right) \cdot\left(\mu_{2} \nabla^{-1}\right) \cdot\left(\mu_{3} \nabla^{-1}\right) \cdot \mu_{4} \cdot \mu_{5} \cdot\left(\mu_{6} \nabla^{-1}\right) \cdot \mu_{7} \\
& =a_{3}^{-1} \cdot\left(a_{3} a_{1}\right)^{-1} \cdot a_{1}^{-1} \cdot a_{3} \cdot a_{2} \cdot a_{1}^{-1} \cdot a_{2}=: \widetilde{\nu}_{1} \\
= & \left(\mu_{1} \nabla^{-1}\right) \cdot \mu_{2} \cdot\left(\mu_{3} \nabla^{-1}\right) \cdot\left(\mu_{4} \nabla^{-1}\right) \cdot \mu_{5} \cdot\left(\mu_{6} \nabla^{-1}\right) \cdot \mu_{7} \\
& =a_{3}^{-1} \cdot a_{2} \cdot a_{1}^{-1} \cdot\left(a_{1} a_{2}\right)^{-1} \cdot a_{2} \cdot a_{1}^{-1} \cdot a_{2}=: \widetilde{\nu}_{2} \\
= & \left(\mu_{1} \nabla^{-1}\right) \cdot \mu_{2} \cdot\left(\mu_{3} \nabla^{-1}\right) \cdot \mu_{4} \cdot\left(\mu_{5} \nabla^{-1}\right) \cdot\left(\mu_{6} \nabla^{-1}\right) \cdot \mu_{7} \\
& =a_{3}^{-1} \cdot a_{2} \cdot a_{1}^{-1} \cdot a_{3} \cdot\left(a_{3} a_{1}\right)^{-1} \cdot a_{1}^{-1} \cdot a_{2}=: \widetilde{\nu}_{3} \\
= & \left(\mu_{1} \nabla^{-1}\right) \cdot \mu_{2} \cdot\left(\mu_{3} \nabla^{-1}\right) \cdot \mu_{4} \cdot \mu_{5} \cdot\left(\mu_{6} \nabla^{-1}\right) \cdot\left(\mu_{7} \nabla^{-1}\right) \\
& =a_{3}^{-1} \cdot a_{2} \cdot a_{1}^{-1} \cdot a_{3} \cdot a_{2} \cdot a_{1}^{-1} \cdot\left(a_{3} a_{1}\right)^{-1}=: \widetilde{\nu}_{4} .
\end{aligned}
$$

We have $(\operatorname{Pos}(g), \operatorname{Neg}(g))=(1,2)$ and $\mathrm{CFB}_{g}=\{(1,2,3,6),(1,3,4,6),(1,3,5,6)$, $(1,3,6,7)\}$. The four distinct geodesic representatives, $\widetilde{\nu}_{1}, \widetilde{\nu}_{2}, \widetilde{\nu}_{3}$ and $\widetilde{\nu}_{4}$, correspond to $(1,2,3,6),(1,3,4,6),(1,3,5,6)$ and $(1,3,6,7)$, respectively. The first word, $\widetilde{\nu}_{1}$, is the geodesic representative chosen in Proposition 3.9:

$$
\begin{aligned}
\eta & \equiv \xi^{(1)} \cdot \lambda \cdot \xi^{(2)} \cdot \mu \cdot \xi^{(3)} \\
& \equiv a_{3}^{-1} \cdot\left(a_{3} a_{1}\right)^{-1} \cdot a_{1}^{-1} \cdot a_{3} \cdot\left(a_{2} \cdot a_{1}^{-1} \cdot a_{2}\right) \equiv \widetilde{\nu}_{1} .
\end{aligned}
$$

\section{$\S 4$. Automata for geodesic representatives of $P_{I_{2}(k)}$}

In this section, we construct deterministic, finite-state automata over subsets of $\mathrm{FB}^{+} \cup \mathrm{FB}^{-} \cup\left\{\nabla, \nabla^{-}\right\}$that recognize a unique geodesic representative for each 
element of $P_{I_{2}(k)}$ specified in $\S 3$. (Refer to $[\mathrm{K}]$ for general information on automata.) As a result, it becomes clear that the spherical growth series for $P_{I_{2}(k)}$ with respect to the generating set $A$ has a rational function expression (cf. [E], [E-IF-Z], [M-M] and $[\mathrm{Sab}])$.

First, we consider all of the geodesic representatives for elements of $P_{I_{2}(k)}$. Here, we define

$$
\begin{aligned}
\widetilde{\boldsymbol{\Gamma}} & :=\left\{\xi \in A^{*}|| \xi \mid=\|\pi(\xi)\|\right\}, \\
\widetilde{\boldsymbol{\Gamma}}_{P, N} & :=\{\xi \in \mathrm{WT}|| \xi \mid=\|\pi(\xi)\|,(\operatorname{Pos}(\xi), \operatorname{Neg}(\xi))=(P, N)\}, \\
G_{P, N} & :=\left\{g \in P_{I_{2}(k)} \mid(\operatorname{Pos}(g), \operatorname{Neg}(g))=(P, N)\right\},
\end{aligned}
$$

where $0 \leq P \leq k$ and $0 \leq N \leq k$. Then, by Propositions 3.3, 3.4 and 3.7 and Corollary 3.8, we have

$$
\begin{aligned}
& P+N \geq k+1 \Rightarrow \widetilde{\boldsymbol{\Gamma}}_{P, N}=\emptyset, \quad G_{P, N}=\emptyset \\
& P+N \leq k \quad \Rightarrow \pi^{-1}\left(G_{P, N}\right) \cap \widetilde{\boldsymbol{\Gamma}}=\widetilde{\boldsymbol{\Gamma}}_{P, N}
\end{aligned}
$$

and

$$
\begin{aligned}
\widetilde{\boldsymbol{\Gamma}} & =\bigcup_{P+N \leq k} \widetilde{\boldsymbol{\Gamma}}_{P, N} \quad \text { (disjoint union) }, \\
P_{I_{2}(k)} & =\bigcup_{P+N \leq k} G_{P, N} \quad \text { (disjoint union). }
\end{aligned}
$$

For each $(P, N)$, the results of Propositions 3.3 and 3.7 are as follows:

1. Case $(P, N)=(k, 0): \pi^{-1}(g) \cap \widetilde{\boldsymbol{\Gamma}}=\mathrm{M} \nabla_{g}^{+}$and $\# \mathrm{M} \nabla_{g}^{+} \geq 2$ for each $g \in G_{k, 0}$;

2. Case $(P, N)=(0, k): \pi^{-1}(g) \cap \widetilde{\boldsymbol{\Gamma}}=\mathrm{M} \nabla_{g}^{-}$and $\# \mathrm{M} \nabla_{g}^{-} \geq 2$ for each $g \in G_{0, k}$;

3. Case $P+N \leq k$ and $(P, N) \notin\{(k, 0),(0, k)\}: \pi^{-1}(g) \cap \widetilde{\boldsymbol{\Gamma}}=\mathrm{SS}_{g}$ for each $g \in G_{P, N}$;

4. Case $P+N \leq k-1: \# \mathrm{SS}_{g}=1$ for each $g \in G_{P, N}$;

5. Case $P+N=k$ and $(P, N) \notin\{(k, 0),(0, k)\}: \# \mathrm{SS}_{g} \geq 2$ for each $g \in G_{P, N}$.

Next, we define a subset $\boldsymbol{\Gamma}_{P, N}$ of $\widetilde{\boldsymbol{\Gamma}}_{P, N}$ such that every element $g$ of $G_{P, N}$ has a unique geodesic representative in $\boldsymbol{\Gamma}_{P, N}$.

[Case 1: $(P, N)=(k, 0)]$ Here, for each $g \in G_{k, 0}$, we choose the normal form $\nu$ of $g$. Then, we define

$$
\boldsymbol{\Gamma}_{k, 0}:=\left\{\begin{array}{l|l}
\nu \in\left(\mathrm{FB}^{+} \cup\{\nabla\}\right)^{*} & \begin{array}{l}
\nu \equiv \mu_{1} \cdots \mu_{m} \cdot \nabla^{d}, \mu_{1} \cdots \mu_{m} \in\left(\mathrm{FB}^{+}\right)^{*} \\
d \geq 1, \text { and } \mathcal{N}\left(\mu_{j}\right) \neq \mathcal{L}\left(\mu_{j+1}\right) \text { for } 1 \leq{ }^{\forall} j \leq m-1
\end{array}
\end{array}\right\}
$$


[Case 2: $(P, N)=(0, k)]$ Here, for each $g \in G_{0, k}$, consider the normal form $\nu$ of $g^{-1} \in G_{k, 0}$. Then, we define

$\Gamma_{0, k}$

$:=\left\{\begin{array}{l|l}\nu^{-1} \in\left(\mathrm{FB}^{-} \cup\left\{\nabla^{-1}\right\}\right)^{*} & \begin{array}{l}\nu \equiv \mu_{1} \cdots \mu_{m} \cdot \nabla^{d}, \mu_{1} \cdots \mu_{m} \in\left(\mathrm{FB}^{+}\right)^{*}, \\ d \geq 1, \text { and } \mathcal{N}\left(\mu_{j}\right) \neq \mathcal{L}\left(\mu_{j+1}\right) \text { for } 1 \leq{ }^{\forall} j \leq m-1\end{array}\end{array}\right\}$.

[Case 3: $P+N \leq k-1]$ In this case, $\mathrm{SS}_{g}$ consists of a single element. Hence, we define

$$
\boldsymbol{\Gamma}_{P, N}:=\widetilde{\boldsymbol{\Gamma}}_{P, N}
$$

Here, introducing simpler notation, we express each element $\xi$ of $\boldsymbol{\Gamma}_{P, N}$ as follows:

$$
\xi \equiv v_{1} \cdots v_{m} \in\left(\mathrm{FB}_{\leq P}^{+} \cup \mathrm{FB}_{\leq N}^{-}\right)^{*},
$$

where for each $j \in\{1, \ldots, m-1\}$, we have

$$
\left\{\begin{array}{l}
v_{j}, v_{j+1} \in \mathrm{FB}^{ \pm} \Rightarrow \mathcal{N}\left(v_{j}\right) \neq \mathcal{L}\left(v_{j+1}\right) \\
v_{j} \in \mathrm{FB}^{ \pm}, v_{j+1} \in \mathrm{FB}^{\mp} \Rightarrow \mathcal{R}\left(v_{j}\right) \neq \mathcal{L}\left(v_{j+1}\right)
\end{array}\right.
$$

and we have

$$
\operatorname{Pos}(\xi)=P, \quad \operatorname{Neg}(\xi)=N
$$

Then, $\boldsymbol{\Gamma}_{P, N}$ is given by

$$
\boldsymbol{\Gamma}_{P, N}=\left\{\xi \in\left(\mathrm{FB}_{\leq P}^{+} \cup \mathrm{FB}_{\leq N}^{-}\right)^{*} \mid \xi \text { is a word as in (30) with (31) and (32) }\right\} .
$$

[Case 4: $P+N=k$, and $(P, N) \notin\{(k, 0),(0, k)\}]$ In this case, although we have $\# \mathrm{SS}_{g} \geq 2$ for each $g \in G_{P, N}$, a unique geodesic representative $\eta$ of $g$ can be specified by Proposition 3.9. Then, we define

$$
\boldsymbol{\Gamma}_{P, N}:=\left\{\eta \in\left(\mathrm{FB}_{\leq P}^{+} \cup \mathrm{FB}_{\leq N}^{-}\right)^{*} \mid \eta \text { satisfies }(24)-(27)\right\} .
$$

Now, with the above, we define $\boldsymbol{\Gamma}$ to be the following disjoint union:

$$
\boldsymbol{\Gamma}:=\boldsymbol{\Gamma}_{k, 0} \cup \boldsymbol{\Gamma}_{0, k} \cup \bigcup_{P+N \leq k-1} \boldsymbol{\Gamma}_{P, N} \cup \underset{\begin{array}{c}
P+N=k \\
(P, N) \neq(k, 0),(0, k)
\end{array}}{\bigcup_{P, N} .}
$$

Since every element of $P_{I_{2}(k)}$ has a unique geodesic representative in $\boldsymbol{\Gamma}$, the restriction of $\pi$ to $\boldsymbol{\Gamma}$ is a bijective map to $P_{I_{2}(k)}$. We call a word in $\boldsymbol{\Gamma}$ the geodesic normal form of the corresponding element of $P_{I_{2}(k)}$.

We now proceed to construct automata that recognize all of the words in $\boldsymbol{\Gamma}$. This is done separately in each of the cases considered above. 
[Case 1: $(P, N)=(k, 0)]$ It is clear that every word in the set $\boldsymbol{\Gamma}_{k, 0}$ is recognized by the deterministic, finite-state automaton $\mathbf{A}_{k, 0}$ over $\mathrm{FB}^{+} \cup\{\nabla\}$ defined by

(i) States: $\{\varepsilon\} \cup \mathrm{FB}^{+} \cup\{\nabla\}$;

Initial state: $\{\varepsilon\}$; Accept state: $\{\nabla\}$;

(ii) Transitions:

$$
\begin{aligned}
& \text { (ii-1) }{ }^{\forall} v \in \mathrm{FB}^{+} \cup\{\nabla\}, \varepsilon \stackrel{v}{\rightarrow} v ; \\
& \text { (ii-2) } \begin{aligned}
& \forall \\
& u, v \in \mathrm{FB}^{+}, u \stackrel{v}{\rightarrow} v \\
& \text { if } \mathcal{N}(u) \neq \mathcal{L}(v) ;
\end{aligned} \\
& \text { (ii-3) }{ }^{\forall} u \in \mathrm{FB}^{+} \cup\{\nabla\}, u \stackrel{\nabla}{\rightarrow} \nabla .
\end{aligned}
$$

[Case 2: $(P, N)=(0, k)]$ For each word in $\boldsymbol{\Gamma}_{0, k}$, its inverse is recognized by the automaton $\mathbf{A}_{k, 0}$ constructed in the previous case. Hence, we need not construct a new automaton in this case.

[Case 3: $P+N \leq k-1]$ It is readily seen that every word in the set $\bigcup_{p \leq P, n \leq N} \boldsymbol{\Gamma}_{p, n}$ is recognized by the deterministic, finite-state automaton $\mathbf{A}_{\leq P, \leq N}$ over $\mathrm{FB}_{\leq P}^{+} \cup$ $\mathrm{FB}_{\leq N}^{-}$defined as follows:

(i) States: $\{\varepsilon\} \cup \mathrm{FB}_{\leq P}^{+} \cup \mathrm{FB}_{\leq N}^{-}$;

Initial state: $\{\varepsilon\} ;$ Accept states: $\{\varepsilon\} \cup \mathrm{FB}_{\leq P}^{+} \cup \mathrm{FB}_{\leq N}^{-}$

(ii) Transitions:

$$
\begin{aligned}
& \text { (ii-1) }{ }^{\forall} v \in \mathrm{FB}_{\leq P}^{+} \cup \mathrm{FB}_{\leq N}^{-}, \varepsilon \stackrel{v}{\rightarrow} v ; \\
& \text { (ii-2) }{ }^{\forall} u, v \in \mathrm{FB}_{\leq P}^{+}, u \stackrel{v}{\rightarrow} v \\
& \text { if } \mathcal{N}(u) \neq \mathcal{L}(v) ; \\
& \text { (ii-3) }{ }^{\forall} u, v \in \mathrm{FB}_{\leq N}^{-}, u \stackrel{v}{\rightarrow} v \text { if } \mathcal{N}(u) \neq \mathcal{L}(v) ; \\
& \text { (ii-4) }{ }^{\forall} u \in \mathrm{FB}_{\leq P}^{+},{ }^{\forall} v \in \mathrm{FB}_{\leq N}^{-}, u \stackrel{v}{\rightarrow} v \\
& \text { if } \mathcal{R}(u) \neq \mathcal{L}(v) ; \\
& \text { (ii-5) }{ }^{\forall} u \in \mathrm{FB}_{\leq N}^{-},{ }^{\forall} v \in \mathrm{FB}_{\leq P}^{+}, u \stackrel{v}{\rightarrow} v \\
& \text { if } \mathcal{R}(u) \neq \mathcal{L}(v) .
\end{aligned}
$$

[Case 4: $P+N=k$, and $(P, N) \notin\{(k, 0),(0, k)\}]$ For each $p$ and $n$ satisfying $p+n \leq k-1$, let $\widehat{\mathbf{A}}_{\leq p, \leq n}$ be the labeled directed graph obtained from $\mathbf{A}_{\leq p, \leq n}$ by deleting the state $\{\varepsilon\}$ and the outgoing transitions from $\{\varepsilon\}$. Let $\mathbf{B}_{0, N}\left(\operatorname{resp} . \mathbf{B}_{P, 0}\right)$ be the graph consisting of $k$ isolated nodes labeled by the elements of $\mathrm{FB}_{N}^{-}$(resp. $\mathrm{FB}_{P}^{+}$). Combining the graphs $\widehat{\mathbf{A}}_{\leq P-1, \leq N-1}, \widehat{\mathbf{A}}_{\leq P-1, \leq N}$ and $\widehat{\mathbf{A}}_{\leq P, \leq N-1}$, linked at $\mathbf{B}_{0, N}$ and $\mathbf{B}_{P, 0}$, we construct the deterministic, finite-state automaton $\mathbf{A}_{P, N}$ over $\mathrm{FB}_{\leq P}^{+} \cup \mathrm{FB}_{\leq N}^{-}$depicted in Figure 1, which recognizes all of the geodesic normal forms in $\boldsymbol{\Gamma}_{P, N}$ : 
(i) States: $\{\varepsilon\} \cup\left\{\right.$ vertices of $\left.\widehat{\mathbf{A}}_{\leq P-1, \leq N-1}\right\} \cup\left\{\right.$ nodes of $\left.\mathbf{B}_{0, N}\right\}$

$\cup\left\{\right.$ vertices of $\left.\widehat{\mathbf{A}}_{\leq P-1, \leq N}\right\} \cup\left\{\right.$ nodes of $\left.\mathbf{B}_{P, 0}\right\} \cup$ vertices of $\left.\widehat{\mathbf{A}}_{\leq P, \leq N-1}\right\}$; Initial state: $\{\varepsilon\}$; Accept states: $\left\{\right.$ nodes of $\left.\mathbf{B}_{P, 0}\right\} \cup$ vertices of $\left.\widehat{\mathbf{A}}_{\leq P, \leq N-1}\right\}$;

(ii) Transitions:

(ii-1) Between the initial state and the outgoing blocks:

${ }^{\forall} v \in\left\{\right.$ vertices of $\left.\widehat{\mathbf{A}}_{\leq P-1, \leq N-1}\right\} \cup\left\{\right.$ nodes of $\left.\mathbf{B}_{0, N}\right\}, \varepsilon \stackrel{v}{\rightarrow} v ;$

(ii-2) Inside of $\widehat{\mathbf{A}}_{\leq P-1, \leq N-1}, \widehat{\mathbf{A}}_{\leq P-1, \leq N}$ and $\widehat{\mathbf{A}}_{\leq P, \leq N-1}$ :

The transitions are represented by the directed edges that come from the original automata $\mathbf{A}_{\leq P-1, \leq N-1}, \mathbf{A}_{\leq P-1, \leq N}$ and $\mathbf{A}_{\leq P, \leq N-1}$;

(ii-3) Between the other blocks:

(ii-3-1) Between $\widehat{\mathbf{A}}_{\leq P-1, \leq N-1}$ and $\mathbf{B}_{0, N}$ :

(ii-3-1-1) ${ }^{\forall} u \in\left\{\right.$ vertices of $\left.\widehat{\mathbf{A}}_{\leq P-1, \leq N-1}\right\} \cap \mathrm{FB}^{+},{ }^{\forall} v \in$ nnodes of $\left.\mathbf{B}_{0, N}\right\}$, $u \stackrel{v}{\rightarrow} v$ if $\mathcal{R}(u) \neq \mathcal{L}(v)$

(ii-3-1-2) ${ }^{\forall} u \in\left\{\right.$ vertices of $\left.\widehat{\mathbf{A}}_{\leq P-1, \leq N-1}\right\} \cap \mathrm{FB}^{-},{ }^{\forall} v \in$ nodes of $\left.\mathbf{B}_{0, N}\right\}$, $u \stackrel{v}{\rightarrow} v$ if $\mathcal{N}(u) \neq \mathcal{L}(v)$

(ii-3-2) Between $\mathbf{B}_{0, N}$ and $\mathbf{B}_{P, 0}:{ }^{\forall} u \in\left\{\right.$ nodes of $\left.\mathbf{B}_{0, N}\right\},{ }^{\forall} v \in$ nodes of $\mathbf{B}_{P, 0}$, $u \stackrel{v}{\rightarrow} v$ if $\mathcal{R}(u) \neq \mathcal{L}(v)$

(ii-3-3) Between $\mathbf{B}_{0, N}$ and $\widehat{\mathbf{A}}_{\leq P-1, \leq N}$ :

(ii-3-3-1) ${ }^{\forall} u \in\left\{\right.$ nodes of $\left.\mathbf{B}_{0, N}\right\},{ }^{\forall} v \in\left\{\right.$ vertices of $\left.\widehat{\mathbf{A}}_{\leq P-1, \leq N}\right\} \cap \mathrm{FB}^{+}, u \stackrel{v}{\rightarrow} v$ if $\mathcal{R}(u) \neq \mathcal{L}(v)$

(ii-3-3-2) ${ }^{\forall} u \in\left\{\right.$ nodes of $\left.\mathbf{B}_{0, N}\right\},{ }^{\forall} v \in\left\{\right.$ vertices of $\left.\widehat{\mathbf{A}}_{\leq P-1, \leq N}\right\} \cap \mathrm{FB}^{-}, u \stackrel{v}{\rightarrow} v$ if $\mathcal{N}(u) \neq \mathcal{L}(v)$

(ii-3-4) Between $\widehat{\mathbf{A}}_{\leq P-1, \leq N}$ and $\mathbf{B}_{P, 0}$ :

(ii-3-4-1) ${ }^{\forall} u \in\left\{\right.$ vertices of $\left.\widehat{\mathbf{A}}_{\leq P-1, \leq N}\right\} \cap \mathrm{FB}^{+},{ }^{\forall} v \in\left\{\right.$ nodes of $\left.\mathbf{B}_{P, 0}\right\}, u \stackrel{v}{\rightarrow} v$ if $\mathcal{N}(u) \neq \mathcal{L}(v)$

(ii-3-4-2) ${ }^{\forall} u \in\left\{\right.$ vertices of $\left.\widehat{\mathbf{A}}_{\leq P-1, \leq N}\right\} \cap \mathrm{FB}^{-},{ }^{\forall} v \in\left\{\right.$ nodes of $\left.\mathbf{B}_{P, 0}\right\}, u \stackrel{v}{\rightarrow} v$ if $\mathcal{R}(u) \neq \mathcal{L}(v)$

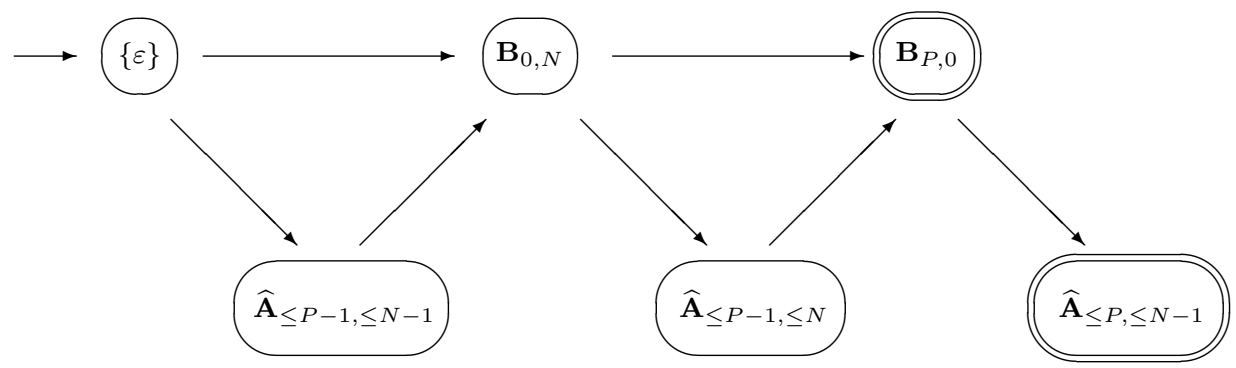

Figure 1. The automaton $\mathbf{A}_{P, N}(P+N=k,(P, N) \notin\{(k, 0),(0, k)\})$. 
(ii-3-5) Between $\mathbf{B}_{P, 0}$ and $\widehat{\mathbf{A}}_{\leq P, \leq N-1}$ :

(ii-3-5-1) ${ }^{\forall} u \in\left\{\right.$ nodes of $\left.\mathbf{B}_{P, 0}\right\},{ }^{\forall} v \in\left\{\right.$ vertices of $\left.\widehat{\mathbf{A}}_{\leq P, \leq N-1}\right\} \cap \mathrm{FB}^{+}, u \stackrel{v}{\rightarrow} v$ if $\mathcal{N}(u) \neq \mathcal{L}(v)$

(ii-3-5-2) ${ }^{\forall} u \in\left\{\right.$ nodes of $\left.\mathbf{B}_{P, 0}\right\},{ }^{\forall} v \in\left\{\right.$ vertices of $\left.\widehat{\mathbf{A}}_{\leq P, \leq N-1}\right\} \cap \mathrm{FB}^{-}, u \stackrel{v}{\rightarrow} v$ if $\mathcal{R}(u) \neq \mathcal{L}(v)$.

\section{§5. Spherical growth series for $P_{I_{2}(k)}$}

In this section, considering the structure of the automata constructed in $\S 4$, we derive an explicit rational function expression for the spherical growth series $\mathcal{S}_{P_{I_{2}(k)}}(t)$ with respect to the generating set $A$.

For each pair $(P, N)$, let

$$
\mathcal{S}_{P, N}(t):=\sum_{q=0}^{\infty} \sharp\left\{\xi \in \boldsymbol{\Gamma}_{P, N}|| \xi \mid=q\right\} t^{q}
$$

be the spherical growth series for $\Gamma_{P, N}$. Then, from the partition (33), we have

$$
\mathcal{S}_{P_{I_{2}(k)}}(t)=\mathcal{S}_{k, 0}(t)+\mathcal{S}_{0, k}(t)+\sum_{P+N \leq k-1} \mathcal{S}_{P, N}(t)+\sum_{\substack{P+N=k \\(P, N) \neq(k, 0),(0, k)}} \mathcal{S}_{P, N}(t)
$$

In order to simplify the presentation of the growth series, for each $q \in \mathbb{N} \cup\{0\}$, we introduce the following:

$$
\left\{\begin{array}{l}
T_{q}:=t+t^{2}+\cdots+t^{q} \quad \text { for } q \geq 1 \\
T_{0}:=0
\end{array}\right.
$$

First, consider the case $P+N \leq k-1$. In this case, we obtain

Proposition 5.1. For each $P, N$ satisfying $P+N \leq k-1$, we have

$$
\sum_{\substack{0 \leq p \leq P \\ 0 \leq n \leq N}} \mathcal{S}_{p, n}(t)=\frac{1+T_{P}+T_{N}}{1-(k-1)\left(T_{P}+T_{N}\right)}
$$

Proof. Choose any $P \in\{0, \ldots, k-1\}$ and $N \in\{0, \ldots, k-1\}$ with $P+N \leq k-1$. For $q \in \mathbb{N} \cup\{0\}$, we define

$$
\begin{aligned}
& B_{q}(P ; N):=\left\{\xi \equiv v_{1} \cdots v_{m} \in \bigcup_{\substack{0 \leq p \leq P \\
0 \leq n \leq N}} \Gamma_{p, n}|| \xi \mid=q\right\}, \\
& \beta_{q}(P ; N):=\sharp B_{q}(P ; N) .
\end{aligned}
$$


Then

$$
\sum_{\substack{0 \leq p \leq P \\ 0 \leq n \leq N}} \mathcal{S}_{p, n}(t)=\sum_{q=0}^{\infty} \beta_{q}(P ; N) t^{q} .
$$

Further, note that for $q=0$, we have

$$
\beta_{0}(P ; N)=1
$$

Also, it is obvious that the result holds for $(P, N)=(0,0)$.

Lemma 5.2. We have the following recursive formulas for $\beta_{q}(P ; N)$ :

(i) If $P \neq 0$ and $N \neq 0$, then

$$
\begin{aligned}
\beta_{q}(P ; N)= & (k-1)\left\{\beta_{q-1}(P ; N)+\cdots+\beta_{q-P}(P ; N)\right\} \\
& +(k-1)\left\{\beta_{q-1}(P ; N)+\cdots+\beta_{q-N}(P ; N)\right\},
\end{aligned}
$$

where $q \geq \max \{P, N\}+1$.

(ii) If $N=0$ and $P \geq 1$ (resp. $P=0$ and $N \geq 1$ ), then

$$
\begin{aligned}
& \beta_{q}(P ; 0)=(k-1)\left\{\beta_{q-1}(P ; 0)+\cdots+\beta_{q-P}(P ; 0)\right\}, \\
& \left(\operatorname{resp} . \beta_{q}(0 ; N)=(k-1)\left\{\beta_{q-1}(0 ; N)+\cdots+\beta_{q-N}(0 ; N)\right\}\right),
\end{aligned}
$$

where $q \geq P+1($ resp. $q \geq N+1)$.

Proof. (i) Employing the automaton $\mathbf{A}_{\leq P, \leq N}$, we observe the following:

- Let $I \in\{1, \ldots, P\}$. For each $v_{1} \cdots v_{m-1} \in B_{q-I}(P ; N)$, from (ii-2) and (ii-5) of $\mathbf{A}_{\leq P, \leq N}$, we have $k-1$ choices of $v_{m} \in \mathrm{FB}_{I}^{+}$such that $v_{1} \cdots v_{m-1} \cdot v_{m}$ is in $B_{q}(P ; N)$.

- Let $J \in\{1, \ldots, N\}$. For each $v_{1} \cdots v_{m-1} \in B_{q-J}(P ; N)$, from (ii-3) and (ii-4) of $\mathbf{A}_{\leq P, \leq N}$, we have $k-1$ choices of $v_{m} \in \mathrm{FB}_{J}^{-}$such that $v_{1} \cdots v_{m-1} \cdot v_{m}$ is in $B_{q}(P ; N)$.

Thus, we obtain the recursive formula appearing in (36).

(ii) Employing the automaton $\mathbf{A}_{\leq P, 0}$ (resp. $\mathbf{A}_{0, \leq N}$ ), we obtain the recursive formula appearing in (37) in the same manner.

Now, we consider several beginning coefficients $\beta_{q}(P ; N)$ for each pair $(P, N)$.

First, consider the cases $N=0, P \neq 0$ and $P=0, N \neq 0$. In these cases, we have

$$
\begin{cases}\beta_{q}(P ; 0)=k^{q} & \text { for } 1 \leq q \leq P \\ \beta_{q}(0 ; N)=k^{q} & \text { for } 1 \leq q \leq N\end{cases}
$$


Thus, using the recursive formula (37) with (35) and (38), we deduce

$$
\begin{aligned}
& \left(\sum_{0 \leq p \leq P} \mathcal{S}_{p, 0}(t)\right) \times\left\{1-(k-1)\left(t+t^{2}+\cdots+t^{P}\right)\right\}=1+t+t^{2}+\cdots+t^{P} \\
& \left(\sum_{0 \leq n \leq N} \mathcal{S}_{0, n}(t)\right) \times\left\{1-(k-1)\left(t+t^{2}+\cdots+t^{N}\right)\right\}=1+t+t^{2}+\cdots+t^{N}
\end{aligned}
$$

Hence,

$$
\begin{aligned}
\sum_{0 \leq p \leq P} \mathcal{S}_{p, 0}(t) & =\frac{1+t+t^{2}+\cdots+t^{P}}{1-(k-1)\left(t+t^{2}+\cdots+t^{P}\right)}=\frac{1+T_{P}}{1-(k-1) T_{P}} \\
\sum_{0 \leq n \leq N} \mathcal{S}_{0, n}(t) & =\frac{1+t+t^{2}+\cdots+t^{N}}{1-(k-1)\left(t+t^{2}+\cdots+t^{N}\right)}=\frac{1+T_{N}}{1-(k-1) T_{N}}
\end{aligned}
$$

for each $t$ in a sufficiently small neighborhood of the origin, 0 .

Next, consider the case $P=N=1$. Then

$$
\beta_{1}(1 ; 1)=2 k,
$$

and, from (36), for $q \geq 2$,

$$
\beta_{q}(1 ; 1)=2(k-1) \beta_{q-1}(1 ; 1) .
$$

Hence, from (35) and (39), we obtain

$$
\sum_{\substack{0 \leq p \leq 1 \\ 0 \leq n \leq 1}} \mathcal{S}_{p, n}(t)=\frac{1+2 t}{1-2(k-1) t}=\frac{1+T_{1}+T_{1}}{1-(k-1) T_{1}-(k-1) T_{1}} .
$$

Now, consider the general case, $P, N \geq 1$ with $(P, N) \neq(1,1)$. In this case, we have

Lemma 5.3. Let $P, N \geq 1$ and $(P, N) \neq(1,1)$. Then

(40) $\beta_{q}= \begin{cases}2 k(2 k-1)^{q-1} & \left(1 \leq{ }^{\forall} q \leq \min \{P, N\}\right), \\ 2 k(2 k-1)^{q-1}-k & (q=\min \{P, N\}+1), \\ (2 k-1) \beta_{q-1} & \left(\min \{P, N\}+2 \leq{ }^{\forall} q \leq \max \{P, N\}\right),\end{cases}$

where $\beta_{q}$ represents $\beta_{q}(P ; N)$.

Proof. It is easy to see that the first equality of (40) holds. In the case $P=N$, the second and third equalities do not appear. Thus, we turn to the case $P \neq N$. Here, we consider only the case $N<P$, because the result for $P<N$ can be 
shown similarly. Thus, we have $\min \{P, N\}=N$ and $\max \{P, N\}=P$. In this case, it is readily verified that $\beta_{N}=2 k(2 k-1)^{N-1}$.

Now, let $w \in B_{N}(P, N)$. Then there are $2 k-1$ choices of $a \in A=A^{+} \cup A^{-}$ such that $|w \cdot a|=\|\pi(w \cdot a)\|=N+1$. Among such elements $w \cdot a(a \in A)$, there are $k$ elements that belong to $\mathrm{FB}_{N+1}^{-}$. We do not regard these $k$ elements as elements of $B_{N+1}(P ; N)$. Hence, we obtain the second equality in (40).

Now, let $q$ be an integer satisfying $N+2 \leq q \leq P$. Choose $v \in B_{q-1}(P ; N)$. Then there are $2 k-1$ choices of $a \in A$ such that $|v \cdot a|=\|\pi(v \cdot a)\|=q$. Among such elements $v \cdot a(a \in A)$, there are $\beta_{q-N-1} \times(k-1)$ elements for which $v \cdot a \equiv u_{1} \cdot u_{2}$ and $\left|u_{1} \cdot u_{2}\right|=\left\|\pi\left(u_{1} \cdot u_{2}\right)\right\|=q$ for some $u_{1} \in B_{q-N-1}(P ; N)$ and $u_{2} \in \mathrm{FB}_{N+1}^{-}$. We do not regard these $(k-1) \beta_{q-N-1}$ elements to be elements of $B_{q}(P ; N)$. Hence, we obtain the third equality in (40).

Using (35), (40) and the recursive formula (36), we obtain

$$
\begin{aligned}
\sum_{\substack{0 \leq p \leq P \\
0 \leq n \leq N}} \mathcal{S}_{p, n}(t) & =\frac{1+t+t^{2}+\cdots+t^{P}+t+t^{2}+\cdots+t^{N}}{1-(k-1)\left(t+t^{2}+\cdots+t^{P}\right)-(k-1)\left(t+t^{2}+\cdots+t^{N}\right)} \\
& =\frac{1+T_{P}+T_{N}}{1-(k-1)\left(T_{P}+T_{N}\right)}
\end{aligned}
$$

for each $t$ in a sufficiently small neighborhood of the origin, 0 .

By considering subseries of the above, we obtain

Corollary 5.4. Let $P$ and $N$ be integers such that $P+N \leq k-1$. Then, for each $u \in \mathrm{FB}_{\leq P}^{+}$(resp. $\left.w \in \mathrm{FB}_{\leq N}^{-}\right)$, the spherical growth series for the set

$$
\begin{aligned}
\boldsymbol{\Lambda}_{u} & :=\left\{v_{1} \cdots v_{m} \in \underset{\substack{0 \leq p \leq P \\
0 \leq n \leq N}}{\bigcup} \boldsymbol{\Gamma}_{p, n} \mid v_{1} \equiv u\right\} \\
\left(\operatorname{resp} . \boldsymbol{\Lambda}_{w}:\right. & \left.=\left\{v_{1} \cdots v_{m} \in \underset{\substack{0 \leq p \leq P \\
0 \leq n \leq N}}{\bigcup} \boldsymbol{\Gamma}_{p, n} \mid v_{1} \equiv w\right\}\right)
\end{aligned}
$$

$i s$

$$
\frac{t^{|u|}}{1-(k-1)\left(T_{P}+T_{N}\right)} \quad\left(\operatorname{resp} \cdot \frac{t^{|w|}}{1-(k-1)\left(T_{P}+T_{N}\right)}\right) .
$$

Proof. Let $\mathcal{L}(u)=a_{i}$ and $\mathcal{L}(w)=a_{j}$. Then, we define

$$
\boldsymbol{\Lambda}_{0}:=\left\{\begin{array}{l|l}
v_{1} \cdots v_{m} \in \bigcup_{\substack{0 \leq p \leq P \\
0 \leq n \leq N}} \boldsymbol{\Gamma}_{p, n} & \begin{array}{l}
\text { if } v_{1} \in \mathrm{FB}_{\leq P}^{+}, \text {then } \mathcal{L}\left(v_{1}\right) \neq a_{i} \\
\text { if } v_{1} \in \mathrm{FB}_{\leq N}^{-}, \text {then } \mathcal{L}\left(v_{1}\right) \neq a_{j}
\end{array}
\end{array}\right\}
$$


Replacing (ii-1) of the automaton $\mathbf{A}_{\leq P, \leq N}$ by the new specification

$$
(\mathrm{ii}-1)^{\prime}\left\{\begin{aligned}
(\mathrm{ii}-1-1)^{\prime} & \forall v \in \mathrm{FB}_{\leq P}^{+}, \varepsilon \stackrel{v}{\rightarrow} v \\
& \text { if } \mathcal{L}(v) \neq a_{i} ; \\
(\mathrm{ii}-1-2)^{\prime} & \forall v \in \mathrm{FB}_{\leq N}^{-}, \varepsilon \stackrel{v}{\rightarrow} v \\
& \text { if } \mathcal{L}(v) \neq a_{j},
\end{aligned}\right.
$$

we obtain a deterministic, finite state automaton $\mathbf{A}_{\leq P, \leq N}^{\prime}$ that recognizes all of the words in $\boldsymbol{\Lambda}_{0}$. Thus, the spherical growth series $\mathcal{S}_{\boldsymbol{\Lambda}_{0}}(t)$ for the set $\boldsymbol{\Lambda}_{0}$ has a rational function expression. Hence, we can write

$$
\mathcal{S}_{\Lambda_{0}}(t)=\frac{E(t)}{F(t)},
$$

where $E(t)$ and $F(t)$ are polynominals.

Now, for each $I \in\{1, \ldots, P\}$ and $J \in\{1, \ldots, N\}$, we define

$$
\begin{aligned}
& \boldsymbol{\Lambda}_{I}^{+}:=\left\{v_{1} \cdots v_{m} \in \bigcup_{\substack{0 \leq p \leq P \\
0 \leq n \leq N}} \boldsymbol{\Gamma}_{p, n} \mid v_{1} \in \mathrm{FB}_{I}^{+}, \mathcal{L}\left(v_{1}\right)=a_{i}\right\}, \\
& \boldsymbol{\Lambda}_{J}^{-}:=\left\{v_{1} \cdots v_{m} \in \bigcup_{\substack{0 \leq p \leq P \\
0 \leq n \leq N}} \boldsymbol{\Gamma}_{p, n} \mid v_{1} \in \mathrm{FB}_{J}^{-}, \mathcal{L}\left(v_{1}\right)=a_{j}\right\} .
\end{aligned}
$$

Then $\bigcup_{0 \leq p \leq P, 0 \leq n \leq N} \boldsymbol{\Gamma}_{p, n}$ can be decomposed into the following disjoint union:

$$
\bigcup_{\substack{0 \leq p \leq P \\ 0 \leq n \leq N}} \boldsymbol{\Gamma}_{p, n}=\boldsymbol{\Lambda}_{0} \cup \boldsymbol{\Lambda}_{1}^{+} \cup \cdots \cup \boldsymbol{\Lambda}_{P}^{+} \cup \boldsymbol{\Lambda}_{1}^{-} \cup \cdots \cup \boldsymbol{\Lambda}_{N}^{-} .
$$

Next, we define

$$
\widehat{\boldsymbol{\Lambda}}_{I}^{+}:=\left\{v_{2} \cdots v_{m} \in \bigcup_{\substack{0 \leq p \leq P \\ 0 \leq n \leq N}} \boldsymbol{\Gamma}_{p, n} \mid v_{1} \cdots v_{m} \in \boldsymbol{\Lambda}_{I}^{+}\right\} .
$$

Consider the Cayley graph of $P_{I_{2}(k)}$ over the generating set $A$. It is then seen that the subgraphs corresponding to $\widehat{\boldsymbol{\Lambda}}_{I}^{+}$and $\boldsymbol{\Lambda}_{0}$ are isometric. Thus, the spherical growth series for $\widehat{\boldsymbol{\Lambda}}_{I}^{+}$is identical to that for $\boldsymbol{\Lambda}_{0}$. Hence, the spherical growth series for $\boldsymbol{\Lambda}_{I}^{+}$is equal to $t^{I} \times \mathcal{S}_{\boldsymbol{\Lambda}_{0}}(t)$. Similarly, the spherical growth series for $\boldsymbol{\Lambda}_{J}^{-}$ is equal to $t^{J} \mathcal{S}_{\boldsymbol{\Lambda}_{0}}(t)$. Therefore, from Proposition 5.1 , the equality (41) and the decomposition (42), we obtain

$$
\begin{aligned}
\frac{1+T_{P}+T_{N}}{1-(k-1)\left(T_{P}+T_{N}\right)} & =\frac{E(t)}{F(t)}+t \frac{E(t)}{F(t)}+\cdots+t^{P} \frac{E(t)}{F(t)}+t \frac{E(t)}{F(t)}+\cdots+t^{N} \frac{E(t)}{F(t)} \\
& =\left(1+T_{P}+T_{N}\right) \frac{E(t)}{F(t)} .
\end{aligned}
$$


Consequently,

$$
\frac{E(t)}{F(t)}=\frac{1}{1-(k-1)\left(T_{P}+T_{N}\right)}
$$

and

$$
\mathcal{S}_{\boldsymbol{\Lambda}_{I}^{+}}(t)=\frac{t^{I}}{1-(k-1)\left(T_{P}+T_{N}\right)}, \quad \mathcal{S}_{\boldsymbol{\Lambda}_{J}^{-}}(t)=\frac{t^{J}}{1-(k-1)\left(T_{P}+T_{N}\right)} .
$$

Finally, since $\boldsymbol{\Lambda}_{u}=\boldsymbol{\Lambda}_{|u|}^{+}$and $\boldsymbol{\Lambda}_{w}=\boldsymbol{\Lambda}_{|w|}^{-}$by definition, the result follows.

Now, consider the positive monoid $P_{I_{2}(k)}^{+}$. Following Proposition 2.9, we regard $P_{I_{2}(k)}^{+}$as a subset of $P_{I_{2}(k)}$. Moreover, we identify $P_{I_{2}(k)}^{+}$with $\bigcup_{0 \leq p \leq k} \boldsymbol{\Gamma}_{p, 0}$ (disjoint union). Also, we identify $\bigcup_{0 \leq p \leq k-1} \boldsymbol{\Gamma}_{p, 0}$ with the maximal subset of $P_{I_{2}(k)}^{+}$whose elements contain no positive word $u$ satisfying $u=\nabla$. With these identifications, we consider the growth series for subsets of $P_{I_{2}(k)}^{+}$. Using Proposition 5.1, we can obtain their rational function expressions according to the following (see [F-S 2] for the proof):

Proposition 5.5. Let $D_{k}(t)$ be the polynomial defined by

$$
D_{k}(t):=1-k t+(k-1) t^{k}=(1-t)\left\{1-(k-1) T_{k-1}\right\} .
$$

Then

$$
\mathcal{S}_{k, 0}(t)=\frac{t^{k}}{D_{k}(t)}, \quad \mathcal{S}_{P_{I_{2}(k)}^{+}}(t):=\sum_{0 \leq p \leq k} \mathcal{S}_{p, 0}(t)=\frac{1}{D_{k}(t)}
$$

Next, consider the case $P+N=k$ with $(P, N) \notin\{(k, 0),(0, k)\}$. In this case, we have

Proposition 5.6. Let $P$ and $N$ be integers that satisfy $P+N=k$ and $(P, N) \notin$ $\{(k, 0),(0, k)\}$. Then

$$
\begin{aligned}
& \mathcal{S}_{P, N}(t) \\
& \quad=\frac{k(k-1) t^{k}}{\left\{1-(k-1)\left(T_{P-1}+T_{N-1}\right)\right\}\left\{1-(k-1)\left(T_{P-1}+T_{N}\right)\right\}\left\{1-(k-1)\left(T_{P}+T_{N-1}\right)\right\}} .
\end{aligned}
$$

Proof. Let

$$
\eta \equiv \xi^{(1)} \cdot \lambda \cdot \xi^{(2)} \cdot \mu \cdot \xi^{(3)}
$$

be the geodesic normal form of the form given in Proposition 3.9. Then the subwords $\lambda, \mu, \xi^{(1)}, \xi^{(2)}$ and $\xi^{(3)}$ satisfy the conditions (24)-(27). Fix $\lambda$. Then, for each $J \in\{1, \ldots, N\}$ (resp. $I \in\{1, \ldots, P-1\}$ ), there exists a unique element 
$w_{\lambda} \in \mathrm{FB}_{J}^{-}\left(\right.$resp. $\left.u_{\lambda} \in \mathrm{FB}_{I}^{+}\right)$such that $\mathcal{L}\left(w_{\lambda}\right)=\mathcal{N}(\lambda)\left(\right.$ resp. $\left.\mathcal{L}\left(u_{\lambda}\right)=\mathcal{R}(\lambda)\right)$. Hence, by Corollary 5.4, the spherical growth series for the sets

$$
\left\{v_{1} \cdots v_{m} \in \bigcup_{\substack{0 \leq p \leq P-1 \\ 0 \leq n \leq N}} \boldsymbol{\Gamma}_{p, n} \mid v_{1} \equiv w_{\lambda}\right\}, \quad\left\{v_{1} \cdots v_{m} \in \bigcup_{\substack{0 \leq p \leq P-1 \\ 0 \leq n \leq N}} \boldsymbol{\Gamma}_{p, n} \mid v_{1} \equiv u_{\lambda}\right\}
$$

are

$$
\frac{t^{J}}{1-(k-1)\left(T_{P-1}+T_{N}\right)}, \quad \frac{t^{I}}{1-(k-1)\left(T_{P-1}+T_{N}\right)},
$$

respectively. Also, there are $k-1$ choices for $\mu$ that yield the geodesic normal form (43). Consequently, with Proposition 5.1, the spherical growth series for the set

$$
\left\{\lambda \cdot \xi^{(2)} \cdot \mu \mid \lambda \cdot \xi^{(2)} \cdot \mu\right. \text { is a subword as in (43)\} }
$$

is found to be

$$
\begin{array}{r}
\left(\frac{1+T_{P-1}+T_{N}}{1-(k-1)\left(T_{P-1}+T_{N}\right)}-\frac{T_{P-1}+T_{N}}{1-(k-1)\left(T_{P-1}+T_{N}\right)}\right)(k-1) t^{k} \\
=\frac{(k-1) t^{k}}{1-(k-1)\left(T_{P-1}+T_{N}\right)} .
\end{array}
$$

Then, applying similar arguments to $\xi^{(1)}$ and $\xi^{(3)}$ (and noting that there are $k$ choices of $\lambda$ ), we obtain the result.

With the above preparation, we finally obtain the rational function expression for the spherical growth series of $P_{I_{2}(k)}$ :

Theorem 5.7. The spherical growth series for the pure Artin group $P_{I_{2}(k)}$ of dihedral type with respect to the generating set $A$ has the rational function expression

$$
\begin{aligned}
& \mathcal{S}_{P_{I_{2}(k)}}(t)=\frac{2 t^{k}}{(1-t)\left\{1-(k-1) T_{k-1}\right\}} \\
& +\sum_{p=1}^{k-1} \frac{k(k-1) t^{k}}{\left\{1-(k-1)\left(T_{p-1}+T_{k-1-p}\right)\right\}\left\{1-(k-1)\left(T_{p-1}+T_{k-p}\right)\right\}\left\{1-(k-1)\left(T_{p}+T_{k-1-p}\right)\right\}} \\
& +\sum_{p=0}^{k-1} \frac{1+T_{p}+T_{k-1-p}}{1-(k-1)\left(T_{p}+T_{k-1-p}\right)}-\sum_{p=0}^{k-2} \frac{1+T_{p}+T_{k-2-p}}{1-(k-1)\left(T_{p}+T_{k-2-p}\right)} \\
& =\frac{1+t^{k}}{(1-t)\left\{1-(k-1) T_{k-1}\right\}} \\
& +\sum_{p=1}^{k-1} \frac{k\left(t^{k-p}+t^{p}\right)}{2\left\{1-(k-1)\left(T_{p-1}+T_{k-p}\right)\right\}\left\{1-(k-1)\left(T_{p}+T_{k-1-p}\right)\right\}} .
\end{aligned}
$$


Proof. The first equality is obtained by simply combining Propositions 5.1, 5.5 and 5.6, and using the trick described in Lemma 5.3 of [M-M]. Next, note that

$$
\mathcal{S}_{i, 0}(t)=\mathcal{S}_{0, i}(t)
$$

for each $i \in\{0, \ldots, k\}$. Using (44), we add each term of $\mathcal{S}_{p, n}(t)$ as follows:

$$
\begin{aligned}
& \text { (45) } \mathcal{S}_{P_{I_{2}(k)}}(t) \\
& =\sum_{\substack{P, N \leq 1 \\
P+N=k}}\left\{\mathcal{S}_{P, N}(t)-\sum_{\substack{0 \leq p \leq P-1 \\
0 \leq n \leq N-1}} \mathcal{S}_{p, n}(t)+\frac{1}{2}\left\{\sum_{\substack{0 \leq p \leq P-1 \\
0 \leq n \leq N}} \mathcal{S}_{p, n}(t)+\sum_{\substack{0 \leq p \leq P \\
0 \leq n \leq N-1}} \mathcal{S}_{p, n}(t)\right\}\right\} \\
& +\mathcal{S}_{k, 0}(t)+\sum_{0 \leq p \leq k} \mathcal{S}_{p, 0}(t) .
\end{aligned}
$$

Then, for each $P(\geq 1)$ and $N(\geq 1)$ satisfying $P+N=k$, by Propositions 5.1 and 5.6 , we obtain

$$
\begin{aligned}
\mathcal{S}_{P, N}(t)-\sum_{\substack{0 \leq p \leq P-1 \\
0 \leq n \leq N-1}} \mathcal{S}_{p, n}(t)+\frac{1}{2}\left\{\sum_{\substack{0 \leq p \leq P-1 \\
0 \leq n \leq N}} \mathcal{S}_{p, n}(t)+\sum_{\substack{0 \leq p \leq P \\
0 \leq n \leq N-1}} \mathcal{S}_{p, n}(t)\right\} \\
=\frac{k\left(t^{N}+t^{P}\right)}{2\left\{1-(k-1)\left(T_{P-1}+T_{N}\right)\right\}\left\{1-(k-1)\left(T_{P}+T_{N-1}\right)\right\}} .
\end{aligned}
$$

Finally, using (45) and (46), with Proposition 5.5, we obtain the second equality of the theorem.

\section{Example 5.8.}

$$
\begin{aligned}
\mathcal{S}_{P_{I_{2}(3)}}(t) & =\frac{(1+t)(1-2 t)\left(1+2 t^{2}\right)}{(1-t)(1-4 t)\left(1-2 t-2 t^{2}\right)} \\
& =1+6 t+30 t^{2}+134 t^{3}+570 t^{4}+2370 t^{5}+9722 t^{6}+39546 t^{7}+O\left(t^{8}\right), \\
\mathcal{S}_{P_{I_{2}(4)}}(t) & =\frac{(1+t)\left(1-9 t+15 t^{2}+21 t^{3}-27 t^{4}+27 t^{5}+27 t^{6}+9 t^{7}\right)}{(1-t)\left(1-6 t-3 t^{2}\right)^{2}\left(1-3 t-3 t^{2}-3 t^{3}\right)} \\
& =1+8 t+56 t^{2}+392 t^{3}+2702 t^{4}+18488 t^{5}+125912 t^{6}+854480 t^{7}+O\left(t^{8}\right), \\
\mathcal{S}_{P_{I_{2}(5)}}(t) & =\frac{(1+t)\left(1+2 t+2 t^{2}\right)\left(1-14 t+50 t^{2}-24 t^{3}-16 t^{4}+32 t^{5}+16 t^{7}\right)}{(1-t)\left(1-8 t-8 t^{2}\right)\left(1-8 t-4 t^{2}-4 t^{3}\right)\left(1-4 t-4 t^{2}-4 t^{3}-4 t^{4}\right)} \\
& =1+10 t+90 t^{2}+810 t^{3}+7290 t^{4}+65522 t^{5}+588450 t^{6}+5281730 t^{7}+O\left(t^{8}\right) .
\end{aligned}
$$

\section{References}

[Be] M. Berger, Minimum crossing numbers for 3-braids, J. Phys. A 27 (1994), 6205-6213. Zbl 0849.57003 MR 1306173

[Bi] J. S. Birman, Braids, links, and mapping class groups, Ann. of Math. Stud. 82, Princeton Univ. Press, Princeton, 1975. Zbl 0305.57013 MR 0375281 
[Br] M. Brazil, Monoid growth functions for braid groups, Int. J. Algebra Comput. 1 (1991), 201-205. Zbl 0305.57013 MR 1128012

[B-Sai] E. Brieskorn and K. Saito, Artin-Gruppen und Coxeter-Gruppen, Invent. Math. 17 (1972), 245-271. Zbl 0243.20037 MR 0323910

[Ca] J. W. Cannon, The combinatorial structure of cocompact discrete hyperbolic groups, Geom. Dedicata 16 (1984), 123-148. Zbl 0606.57003 MR 0758901

[Ca-W] J. W. Cannon and Ph. Wagreich, Growth functions of surface groups, Math. Ann. 293 (1992), 239-257. Zbl 0734.57001 MR 1166120

[C1] R. Charney, Artin groups of finite type are biautomatic, Math. Ann. 292 (1992), 671683. Zbl 0736.57001 MR 1157320

[C2] R. Charney, Geodesic automation and growth functions for Artin groups of finite type, Math. Ann. 301 (1995), 307-324. Zbl 0813.20042 MR 1314589

[dlH] P. de la Harpe, Topics in geometric group theory, Chicago Lectures in Math., Univ. of Chicago Press, 2000. Zbl 0965.20025

[D] P. Deligne, Les immeubles des groupes de tresses généralisés, Invent. Math. 17 (1972), 273-302. Zbl 0238.20034 MR 0422673

[E] D. B. A. Epstein with J. W. Cannon, D. F. Holt, S. V. F. Levy, M. S. Paterson and W. P. Thurston, Word processing in groups, Jones and Bartlett, Boston, 1992. Zbl 0764.20017 MR 1161694

[E-IF-Z] D. B. A. Epstein, A. R. Iano-Fletcher and U. Zwick, Growth functions and automatic groups, Experiment. Math. 5 (1996), 297-315. Zbl 0892.20022 MR 1437220

[Fl-P] W. J. Floyd and S. P. Plotnick, Growth functions on Fuchsian groups and the Euler characteristic, Invent. Math. 88 (1987), 1-29. Zbl 0608.20036 MR 0877003

[F-S 1] M. Fujii and T. Satoh, A necessary condition for representatives of elements of Artin groups of dihedral type to be geodesic, Hiroshima Math. J. 43 (2013), 139-147.

[F-S 2] M. Fujii and T. Satoh, The growth series for pure Artin monoids of dihedral type, preprint, 2012

[G] F. Garside, The braid groups and other groups, Quart. J. Math. Oxford 20 (1969), 235-254. Zbl 0194.03303 MR 0248801

[K] D. C. Kozen, Automata and computability, Undergrad. Texts in Comput. Sci., Springer, 1997. Zbl 0883.68055 MR 1633052

[M-M] J. Mairesse and F. Mathéus, Growth series for Artin groups of dihedral type, Int. J. Algebra Comput. 16 (2006), 1087-1107. Zbl 1142.20018 MR 2286424

[Mic] J. Michel, A note on words in braid monoids, J. Algebra 215 (1999), 366-377. Zbl 0937.20017 MR 1684142

[Mil] J. Milnor, A note on curvature and fundamental group, J. Differential Geom. 2 (1968), 1-7. Zbl 0162.25401 MR 0232311

[O] O. Öre, Linear equations in non-commutative fields, Ann. of Math. 32 (1931), 463-477. Zbl 0001.26601 MR 1503010

[R] R. Randell, The fundamental group of the complement of a union of complex hyperplanes, Invent. Math. 69 (1982), 103-108. Zbl 0505.14017 MR 0671654

[Sab] L. Sabalka, Geodesics in the braid group on three strands, in Group theory, statistics and cryptography, Contemp. Math. 360, Amer. Math. Soc., 2004, 133-150. Zbl 1065.20048 MR 2105441

[Sc] A. S. Schwarz, A volume invariant of coverings, Dokl. Akad. Nauk SSSR 105 (1955), 32-34 (in Russian).

[St] M. Stoll, Rational and transcendental growth series for the higher Heisenberg groups, Invent. Math. 126 (1996), 85-109. Zbl 0869.20018 MR 1408557 